\title{
Genotypic and Haplotype analysis of Interleukin-6 and-18 gene polymorphisms in association with Clinicopathological factors in Breast cancer
}

Chiranjeevi Padala ${ }^{1,2}$, Kaushik Puranam ${ }^{1}$, Nivas Shyamala ${ }^{1}$, Keerthi Kupsal ${ }^{1}$, Ramanjaneyulu Kummari ${ }^{1}$, Rajesh Kumar Galimudi ${ }^{3}$, Kishore Kumar Gundapaneni ${ }^{1}$, Mohini Aiyengar Tupurani ${ }^{1}$, Aparna Suryadevera ${ }^{4}$, Sanjeeva Kumari Chinta ${ }^{4}$, Surekha Rani Hanumanth ${ }^{1}$

1. Department of Genetics and Biotechnology, Osmania University, Hyderabad, INDIA

2. Department of Biochemistry, School of Life Sciences, University of Hyderabad, Hyderabad, INDIA

3. MAA Research Foundation, Jubilee Hills, Hyderabad, INDIA

4. MNJ Institute of Oncology and Regional Cancer Center, Hyderabad, INDIA

Simple Summary: The etiology of breast cancer is multifactorial and heterogeneous; it appears to involve numerous genetic/epigenetic, endocrine, and external environmental factors. The IL6 and ILI8 network is highly complex and employs an interactive cascade of gene activation and suppression. The present study performed a comprehensive analysis to understand the role of epidemiological, clinic-pathological characteristics and effect of functional SNPs of IL6 and IL18, in breast cancer patients in comparison to controls. Genotype, haplotype, LD, and survival analysis revealed a significant association between epidemiological, clinical variables and the progression of breast cancer. The data suggest genetic polymorphisms of IL6 (-597G $>$ A, $-572 \mathrm{C}>\mathrm{G} \&-174 \mathrm{G}>\mathrm{C})$ and $I L 18$ genes $(-607 \mathrm{C}>\mathrm{A},-137 \mathrm{G}>\mathrm{C} \& 105 \mathrm{~A}>\mathrm{T})$ may influence the gene transcription, cytokine secretion and thereby modulate the risk for progression of the disease.

\section{Abstract:}

Background: Cytokines are multifunctional glycoproteins playing a vital role in the tumor microenvironment and in the progression of breast cancer. The immune responses in the tumours restrained by pro- and antiinflammatory cytokine expressions could be influenced by genetic polymorphisms. Hence, the present study evaluated the contribution of IL6 (rs1800797, rs1800796, and rs1800795), and IL18 (rs1946518, rs187238, and rs549908) genotypes and its haplotypes with risk and progression of breast cancer in south Indian population.

Methods: The polymorphisms of IL6 gene $-597 \mathrm{G}>\mathrm{A},-572 \mathrm{G}>\mathrm{C},-174 \mathrm{G}>\mathrm{C}$ and IL18 gene $-607 \mathrm{C}>\mathrm{A},-137 \mathrm{G}>\mathrm{C}$, and $105 \mathrm{~A}>\mathrm{T}$ were genotyped through PCR-RFLP and As-PCR assays in blood DNA of 600 subjects. We have performed haplotype, $\mathrm{LD}$, univariate, multivariate logistic regression and Kaplan-Meier analyses for the obtained data. Results: The frequency of AA genotype \& A-allele of IL6 -597G>A, and CC genotype \& C allele of IL6 $174 \mathrm{G}>\mathrm{C}$ polymorphism was higher in breast cancer patients and was found to be significantly associated with TNM staging, late (advanced) stage, metastasis, etc. While, CG and GG genotypes of IL6 -572 C>G polymorphism had a protective role against breast cancer. Further, IL18 -607C $>$ A, $-137 \mathrm{G}>\mathrm{C} \& 105 \mathrm{~A}>\mathrm{T}$ polymorphisms were found to be associated with lobular carcinoma subtype, $\mathrm{PR}^{-\mathrm{ve}}$ and $\mathrm{HER} 2^{+\mathrm{ve}}$ breast cancer patients. Perfect LD was observed between all SNPs of IL6 \& IL18 genes under study; G-C-C, A-G-G and A-C-C haplotype combination of IL6 genes had conferred 2.09, 2.25 and 4.72 folds risk for breast cancer respectively. In 
survival analysis, we observed that the $\mathrm{C}$ allele of rs 1800795 was found to be significantly associated with 5years overall survival in breast cancer subjects. Conclusions: Overall, our results suggest the importance of genotypic and haplotype analysis of IL6, and IL18 gene variants in progression and risk identification of breast cancer.

Keywords: Breast Cancer; Haplotype; Single nucleotide polymorphisms; Interleukin-6 and Interleukin-18.

\section{Introduction}

Breast cancer (BC) a strong interaction between genetic and epigenetic factors cause dynamic changes in the genome leading to uncontrolled cell growth, ability to invade and metastasize [1,2]. Recent experimental evidences implicate the role of cytokines in breast cancer development and progression. Interleukins $(I L)$ are a class of cytokines primarily expressed by leukocytes and are low molecular weight protiens, which act on many target cells often in an additive, synergistic or antagonistic manner and are considered as key intercellular mediators that control survival, growth, differentiation, and the effector cell function. In addition, they are also involved in the pathology of BC surveillance system and other complex diseases [3-5].

IL6 and IL18 belong to the ILI cytokine family and are known to regulate the immune reactions, inflammation and promote tumor growth by up-regulating angiogenic and anti-apoptotic proteins [4]. The genes for $I L 6$ and $I L 18$ are encoded on human chromosome 7p15.3 and 11q23.1 respectively. It has been reported that several important polymorphic sites in the $I L 6$ and $I L 18$ genes, including three in the promoter regions $(-597 \mathrm{G}>\mathrm{A}$, $-572 \mathrm{C}>\mathrm{G} \&-174 \mathrm{G}>\mathrm{C})$ of IL6 and two in promoter and one in exon regions (-607C $>\mathrm{A},-137 \mathrm{G}>\mathrm{C} \& 105 \mathrm{~A}>\mathrm{T})$ of IL18 play an important role in breast cancer progression [6-8]. Furthermore, the single nucleotide polymorphisms (SNPs) have been considered to be important biomarkers in cancer screening, staging, grading and risk assessment of the disease [9].

Despite the limitations in individual SNP analysis, haplotype blocks in the human genome which are formed as a result of linkage disequilibrium, can be used to identify its association with human diseases like cancer [10]. Hence possible that a haplotype effect, rather than an individual effect of SNPs on clinicopathological status, might explain some of the prognostic and survival information. The aim of the present study is to perform a comprehensive analysis of the possible prognostic importance of genotypic and haplotypes of the IL6 gene $(-597 \mathrm{G}>\mathrm{A},-572 \mathrm{C}>\mathrm{G} \&-174 \mathrm{G}>\mathrm{C})$ and $I L 18$ gene $(-607 \mathrm{C}>\mathrm{A},-137 \mathrm{G}>\mathrm{C} \& 105 \mathrm{~A}>\mathrm{T})$ polymorphisms with clinicopathological status in susceptibility and progression of breast cancer. 


\section{Materials and methods}

\subsection{Study Design and Participants}

A total of 300 breast cancer patients and 300 healthy cancer-free control subjects with similar socioeconomic and geographic backgrounds were included in the study after signed written informed consent. The patients with Breast cancer were recruited from the MNJ Institute of Oncology and Regional Cancer Centre (MNJIO\&RCC), Hyderabad during the period of 2010-2016. The histo-clinicopathological information of the patients was obtained from their medical records in the hospital. All the samples were coded and maintained confidentially. The study has been approved by the Institutional and Hospital review boards of Osmania University and MNJIO\&RCC-Hyderabad, Telangana state, INDIA.

\subsection{Polymorphism and SNP Genotyping analysis}

Genomic DNA was extracted from peripheral blood using the Bio-serve DNA Blood Mini kit (Bio-Serve, INDIA), according to the manufacturer's instructions [11]. The genotypes of IL6 (-597G $>\mathrm{A},-572 \mathrm{C}>\mathrm{G}$ and $174 \mathrm{G}>\mathrm{C})$ and $I L 18(-607 \mathrm{C}>\mathrm{A},-137 \mathrm{G}>\mathrm{C}$ and $105 \mathrm{~A}>\mathrm{T})$ gene polymorphisms were determined using PCR-RFLP (Polymerase chain reaction-Restriction Fragment Length Polymorphism) and As-PCR (Allele SpecificPolymerase Chain Reaction) methods respectively. PCR primers, RFLP enzymes, size of the PCR-RFLP products and conditions are shown Table 1. As a genotyping quality assessment purpose, we have randomly selected and repeated $10 \%$ of the samples. The samples were found to be $100 \%$ concordant in two independent assays.

Table 1: Primer sequences and reaction conditions for genotyping $I L 6$ and $I L 18$ polymorphisms

\begin{tabular}{|c|c|c|c|c|}
\hline $\begin{array}{l}\text { Polymorphism } \\
\text { (db SNP ID) }\end{array}$ & PCR Primer sequence & $\begin{array}{l}\mathrm{mT} \\
\left({ }^{\circ} \mathrm{C}\right)\end{array}$ & $\begin{array}{l}\text { Amplicon } \\
\text { Size (bp)/RE }\end{array}$ & $\begin{array}{l}\text { Product size } \\
\text { (bp) }\end{array}$ \\
\hline $\begin{array}{l}\text { IL6 -597G }>\text { A } \\
(\mathrm{rs} 1800797)\end{array}$ & $\begin{array}{l}\text { F: 5'-GGAGTCACACACTCCACCTG-3' } \\
\text { R: 5'-AAGCAGAACCACTCTTCCTTTACTT-3' }\end{array}$ & 61.5 & $\begin{array}{l}527 \\
\text { FokI }\end{array}$ & $\begin{array}{l}\text { GG:527 } \\
\text { GA:527+461+66 } \\
\text { AA: } 461+66\end{array}$ \\
\hline $\begin{array}{l}\text { IL6 -572C }>\text { G } \\
(\mathrm{rs} 1800796)\end{array}$ & $\begin{array}{l}\text { F: 5'-GGAGACGCCTTGAAGTAACTGC-3' } \\
\text { R: 5'-GAGTTTCCTCTGACTCCATCGCAG-3' }\end{array}$ & 55.0 & $\begin{array}{l}296 \\
B s r B I\end{array}$ & $\begin{array}{l}\text { CC: } 296 \\
\text { CG: } 296+201+95 \\
\text { GG:201+95 }\end{array}$ \\
\hline $\begin{array}{l}\text { IL6 -174G >C } \\
(\mathrm{rs} 1800795)\end{array}$ & $\begin{array}{l}\text { F: 5'-ATGCCAAGTGCTGAGTCACTA-3' } \\
\text { R: 5'-TCGAGGGCAGAATGAGCCTC-3' }\end{array}$ & 59.7 & $\begin{array}{l}230 \\
\text { NlaIII }\end{array}$ & $\begin{array}{l}\text { GG: } 230 \\
\text { GC: } 230+121+109 \\
\text { CC- } 121+109\end{array}$ \\
\hline $\begin{array}{l}\text { IL18 -607C }>\text { A } \\
(\mathrm{rs} 1800795)\end{array}$ & $\begin{array}{l}\text { F: 5'-CTTTGCTATCAT TCCAGGAA-3' } \\
\text { R: 5'-TAACCTCATTCAGGACTTCC-3' } \\
\text { C allele:5'- } \\
\text { GTTGCAGAAAGGTAAAAATTATTAC-3' } \\
\text { A allele:5'- } \\
\text { TTGCAGAAAGTGTAAAAATTATTAA-3' }\end{array}$ & 56.5 & $\begin{array}{l}\text { As-PCR } \\
306 \\
196\end{array}$ & $\begin{array}{l}\text { CC:306+196; } 306 \\
\text { CA:306+196;306+196 } \\
\text { AA-306, 306+196 } \\
\text { Common band: } 306 \\
\text { C/A allele: } 196\end{array}$ \\
\hline $\begin{array}{l}\text { IL18 -137G }>C \\
(\mathrm{rs} 187238)\end{array}$ & $\begin{array}{l}\text { F: 5'-CCAATAGGACTGATTATTCCGCA-3' } \\
\text { R:5'-AGGAGGGCAAAATGCACTGG-3' } \\
\text { G allele: 5'- } \\
\text { CCCCAACTTTTACGGAAGAAAAG-3' } \\
\text { C allele: 5'- } \\
\text { CCCCAACTTTTACGGAAGAAAAC-3' }\end{array}$ & 62.5 & $\begin{array}{c}A s-P C R \\
446 \\
261\end{array}$ & $\begin{array}{l}\text { GG: } 446+261 ; 261 \\
\text { GC: } 446+261 ; 446+261 \\
\text { CC: } 446 ; 446+261 \\
\text { Common band- } 446 \\
\text { G/C allele: } 261\end{array}$ \\
\hline
\end{tabular}




\begin{tabular}{|c|c|c|c|c|}
\hline $\begin{array}{l}\text { IL18 105A>T } \\
\text { (rs549908) }\end{array}$ & $\begin{array}{l}\text { F: 5'-TGTTTATTGTAGAAAACCTGGAATT-3' } \\
\text { R: 5'-CCTCTACAGTCAGAATCAGT-3' }\end{array}$ & 50.0 & $\begin{array}{l}148 \\
\text { FokI }\end{array}$ & $\begin{array}{l}\text { AA: } 148, \text { AT: } \\
148+123+25 \\
\text { TT: } 123+25\end{array}$ \\
\hline
\end{tabular}

Abbreviations: dbSNP ID, database identifier; single-nucleotide polymorphism (SNP); polymerase chain reaction (PCR); forward primer $(\mathrm{F})$; reverse primer $(\mathrm{R})$; melting temperature $(\mathrm{mT})$; restriction endonuclease $(\mathrm{RE})$; base pair $(\mathrm{bp})$.

\subsection{Statistical analysis}

$\chi 2$ (chi-squraed) test and the odds ratio (OR) for allele and genotype frequencies of the studied polymorphism between cases and healthy controls were determined. All the $p$ values were two sided, and the level of significance was taken as $p<0.05$. The different statistical tools used to analyse the quantitative and qualitative variables in the present study are IBM SPSS version 20.0, SNPStats (http://bioinfo.iconcologia .net/index.php? module 1/4Snp stats)[12], HAPLOVIEW (http://www.broad.mit.edu/mpg/haploview) [13], Generalized Linkage disequilibrium and Graph Pad Prism [14].

\subsection{Insilico Analysis}

The SNPs in the promoter regions of IL6, and IL18 genes were studied for the presence of transcription factor binding sites using AliBaba2.1 online bioinformatics tool ( $\mathrm{http} / /$ www.gene-regulation. com/pub/programs/alibaba2/index.html). Pre mRNA Secondary structures for IL18 105A>T (exon) were identified by using RNAfold WebServer tool originally proposed by Zuker and Stiegler (http://rna.tbi.Univie.ac.at /cgi-bin/RNAWebSuite/RNAfold.cgi) [15]. Protein-protein interaction (PPI) analysis of IL6 and IL18 was performed using Search tool for the Retrieval of Intracting Genes/Protines (STRING 9.1 http://string.embl.de/) [16].

\section{RESULTS}

\subsection{Comparison of demographic characteristics among patients with breast cancer and controls}

The detailed demographic and baseline clinicopathological characteristics of study subjects are illustrated in Fig. 1. In the present study the age of the breast cancer patients was within a range of 30-75 years, and their average age at diagnosis was 47.98 years $(\mathrm{SD}: \pm 10.80)$ while the age of the controls at recruitment ranged from 35-68 years with a mean age of 46.34 years $(\mathrm{SD}: \pm 7.97)$. An interesting finding that there was a significantly greater proportion of subjects are with higher BMI, mixed diet, history of smoking habit and alcohol consumption in the BC group $(37.0 \%, 85.56 \%, 18.34 \%$ and $40.4 \%)$ than in the carcinoma-free control group $(15.7 \%, 71.0 \%$, $9.0 \%$ and $19.0 \%$ ) respectively ( $p<0.001$ ), while there was a no significant difference observed in age, area of living and occupation $(p>0.05)$ of the patients and controls as shown in Fig.1A. The frequency distributions of age at late menarche $>14$ years $(27.0 \%)$, nulliparous $(8.34 \%)$, lactation $(9.0 \%)$ and history of parental consanguinity (37.0\%) were high in breast cancer patients compared to controls. Moreover, there was no significant difference between the two groups in the frequency distribution in terms of menstrual cycles, and menstruation status 
$(p>0.05)$. The familial incidence of the breast cancer was found to be $15.0 \%$ while the same of the other carcinomas was found to be $19 \%$ in breast cancer patients as shown in Fig.1B.

The clinicopathological characteristics of breast cancer cases are shown in Fig. 1C. In the present study, breast cancer patients were categorized according to tumor node metastasis (TNM) classification for breast cancer and found that $65.67 \%$ are with $\mathrm{T}_{0}-\mathrm{T}_{2}$ stage, $34.33 \%$ are $\mathrm{T}_{3}-\mathrm{T}_{4}$ stages. Among the breast cancer patients $65.7 \%$ have shown about $<50 \mathrm{~mm}$ tumor size. The percentage of histologically confirmed breast cancer patients was $82.33 \%$ in ductal carcinoma and $18.0 \%$ had lobular carcinoma. Whereas, $73.33 \%$ of patients had positive axillary lymph node metastasis and $34.4 \%$ had distant metastasis. According to steroid hormonal clinical characteristics, patients were classified as with positive receptor status for ER (59.0\%), PR (56.0\%,), HER2/neu (54.33\%,) and for some of the patients there was a missing data on receptor status $(8.67 \%)$ respectively.
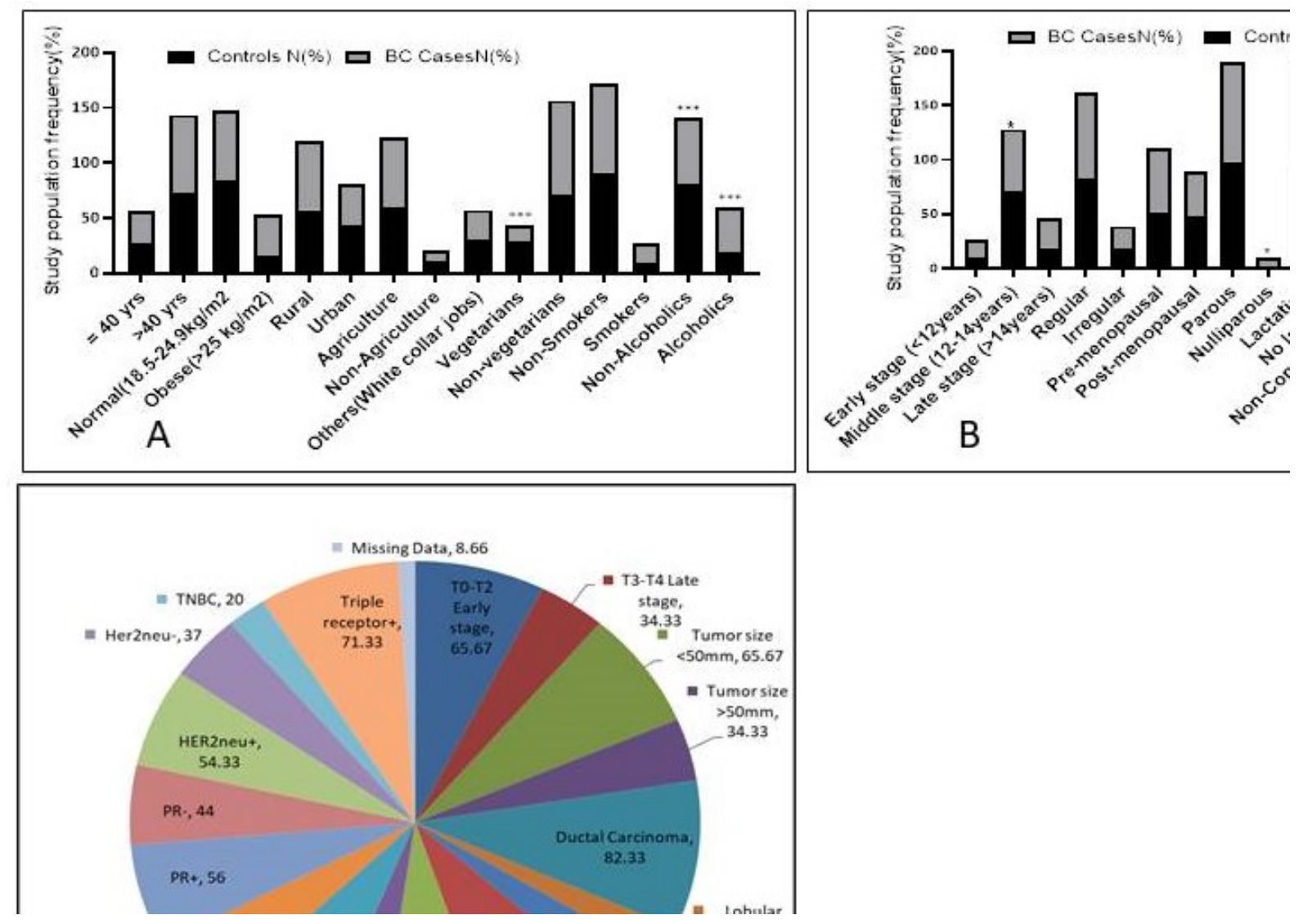

Figure: 1. Distributions of the frequencies study population(Controls N=300 and BC cases N=300): Distributions of the frequencies of epidemiological characteristics controls and $\mathrm{BC}$ cases $\mathrm{A}$, Baseline clinical and family history characteristics $\mathrm{B}$, Clinicopathological risk parameters prevalence in the breast cancer cases $\mathrm{C}$. 


\subsection{Association Analysis of Alleles and Genotypes with Risk of Breast cancer}

The genotypic and allele frequencies of 6 SNPs in the IL6 (-597G>A,-572C $>\mathrm{G},-174 \mathrm{G}>\mathrm{C})$ and IL18 ($607 \mathrm{C}>\mathrm{A},-137 \mathrm{G}>\mathrm{C}, 105 \mathrm{~A}>\mathrm{T}$ ) genes in 300 breast cancer patients and 300 controls groups adjusted with age by multivariate logistic regression analyses are summarized in Table 2. The frequency of A-allele and the GA and AA genotypes of IL6 -597G $>$ A polymorphism have shown a significant association $(p<0.01)$ with breast cancer. The frequency of G-allele and GG genotype of IL6 -572C $>$ G polymorphism was found to be less in breast cancer cases when compared to controls. Further analysis revealed that the C allele of IL6 -174G $>$ C polymorphism was found to be significantly associated with breast cancer patients when compared to the healthy controls.

The genotype frequency of $I L 18-607 \mathrm{C}>\mathrm{A}$ polymorphism did not revealed any significant association with breast cancer . The $\mathrm{C}$-allele of $-137 \mathrm{C}>\mathrm{A}$ gene polymorphism was found to be similar in both controls and breast cancer patients, though the $\mathrm{CC}$ genotype was observed to be significantly associated with risk for development of breast cancer $(p<0.04)$. Further TT genotype frequency of IL18 105A>T polymorphism was marginally higher in breast cancer cases compared to controls are showed in Table 2 . The genotype frequencies of IL18 -607C $>\mathrm{A}$ and IL18 105A $>$ T polymorphisms are following Hardy-Weinberg equilibrium in both controls and breast cancer patients $(p>0.05)$, and the IL6 $-597 \mathrm{G}>\mathrm{A}$, IL6 $-572 \mathrm{C}>\mathrm{G}$, IL6 $-174 \mathrm{G}>\mathrm{C}$ and IL18 $-137 \mathrm{G}>\mathrm{C}$ polymorphisms have deviated from Hardy-Weinberg equilibrium in both controls and cases $(p<0.05)$. The Hardy-Weinberg equilibrium deviation in controls generally reflects the gene pool of the population studied pointing to gene diversity and fixation of alleles in the population.

Table 2: Distribution of $I L 6$ and $I L 18$ genotypes among the breast cancer patients and non-cancer healthy control subjects

\begin{tabular}{|c|c|c|c|c|c|c|c|c|}
\hline \multirow{2}{*}{$\begin{array}{l}\text { Polymorphism } \\
\text { IL6 }-597 \mathrm{G}>\mathrm{A}\end{array}$} & \multicolumn{3}{|c|}{ Genotype frequency } & \multirow{2}{*}{$\begin{array}{l}\begin{array}{l}\text { Allele } \\
\text { frequency }\end{array} \\
\mathrm{G} / \mathrm{A}\end{array}$} & \multirow{2}{*}{$\begin{array}{l}p- \\
\text { value }\end{array}$} & \multirow[t]{2}{*}{ Model } & \multirow{2}{*}{$\begin{array}{l}\text { AOA } \\
\text { OR(95\%CI })\end{array}$} & \multirow{2}{*}{$\begin{array}{l}\chi^{2} \\
p \text {-value }\end{array}$} \\
\hline & GG & GA & AA & & & & & \\
\hline Controls $(n=300)$ & 74.0 & 17.0 & 9.0 & $0.82 / 0.18$ & \multirow[t]{3}{*}{$0.01 *$} & Additive & $2.17(1.70-2.76)$ & $0.01 *$ \\
\hline Cases $(n=300)$ & \multirow{2}{*}{\multicolumn{2}{|c|}{$2.53(1.93-2.32)$}} & \multicolumn{2}{|c|}{$2.53(1.93-2.32)$} & & Co-dominant2 & $3.97(2.38-6.65)$ & \\
\hline $\begin{array}{l}\text { HWE }(p) \\
\text { Control } \\
\text { Cases }\end{array}$ & & & & & & Dominant & $3.23(2.26-4.62)$ & $0.01 *$ \\
\hline IL6 $-572 \mathrm{C}>\mathrm{G}$ & $\mathrm{CC}$ & $\mathrm{CG}$ & GG & $\mathrm{C} / \mathrm{G}$ & & & & \\
\hline \multirow{3}{*}{$\begin{array}{l}\text { Controls }(n=300) \\
\text { Cases }(n=300)\end{array}$} & 17.0 & 59.7 & 23.3 & $0.47 / 0.53$ & \multirow[t]{4}{*}{$0.03 *$} & Additive & $0.67(0.51-0.87)$ & $0.002 *$ \\
\hline & 24.0 & 62.3 & 13.7 & $0.55 / 0.45$ & & Co-dominant1 & $0.79(0.52-1.20)$ & $0.007 *$ \\
\hline & & & OR & $1(0.57-0.89)$ & & Co-dominant2 & $0.44(0.26-0.76)$ & \\
\hline $\begin{array}{l}\text { HWE(p) } \\
\text { Control } \\
\text { Cases }\end{array}$ & & & & & & Dominant & $0.69(0.46-1.04)$ & 0.07 \\
\hline \multirow[t]{2}{*}{ Cases $(n=300)$} & 36.3 & 40.0 & 23. & $0.56 / 0.44$ & & Co-dominant1 & $1.93(1.33-2.29)$ & $0.01 *$ \\
\hline & & & \multicolumn{2}{|c|}{ OR 1.91(1.50-2.43) } & & Co-dominant2 & $2.52(1.59-4.00)$ & \\
\hline $\mathrm{HWE}(\mathrm{p})$ & & & & & & Dominant & $2.11(1.52-2.95)$ & $0.01 *$ \\
\hline
\end{tabular}




\begin{tabular}{|c|c|c|c|c|c|c|c|c|}
\hline Control & 0.01 & & & & & Recessive & $1.89(1.23-2.91)$ & $0.003 *$ \\
\hline Cases & 0.0015 & & & & & Over-dominant & $1.48(1.05-2.08)$ & $0.026^{*}$ \\
\hline IL18 -607C >A & $\mathrm{CC}$ & CA & AA & $\mathrm{C} / \mathrm{A}$ & & & & \\
\hline Controls $(\mathrm{n}=300)$ & 60.0 & 33.7 & 6.3 & $0.77 / 0.23$ & 0.25 & Additive & $1.11(0.85-1.44)$ & 0.45 \\
\hline $\operatorname{Cases}(\mathrm{n}=300)$ & 55.7 & 36.7 & $\begin{array}{l}7.7 \\
\text { OR } 1.1\end{array}$ & $\begin{array}{c}0.74 / 0.26 \\
6(0.89-1.51)\end{array}$ & & $\begin{array}{l}\text { Co-dominant } 1 \\
\text { Co-dominant } 2\end{array}$ & $\begin{array}{l}1.15(0.81-1.63) \\
1.14(0.59-2.21)\end{array}$ & 0.71 \\
\hline HWE(p) & & & & & & Dominant & $1.15(0.83-1.60)$ & 0.41 \\
\hline Control & 0.33 & & & & & Recessive & $1.08(0.57-2.07)$ & 0.81 \\
\hline Cases & 0.45 & & & & & Over-dominant & $1.13(0.81-1.59)$ & 0.47 \\
\hline IL18-137G $>C$ & GG & $\mathrm{GC}$ & $\mathrm{CC}$ & $\mathrm{G}>\mathrm{C}$ & & & & \\
\hline Controls $(\mathrm{n}=300)$ & 49.3 & 43.7 & 7.0 & $0.71 / 0.29$ & 0.94 & Additive & $0.98(0.76-1.26)$ & 0.86 \\
\hline $\operatorname{Cases}(\mathrm{n}=300)$ & 53.7 & 35.3 & $\begin{array}{l}11.0 \\
\text { OR } 0.9\end{array}$ & $\begin{array}{r}0.71 / 0.29 \\
(0.71-1.27)\end{array}$ & & $\begin{array}{l}\text { Co-dominant } 1 \\
\text { Co-dominant } 2\end{array}$ & $\begin{array}{l}0.73(0.51-1.02) \\
1.43(0.78-2.61)\end{array}$ & 0.04 \\
\hline HWE(p) & & & & & & Dominant & $0.82(0.59-1.14)$ & 0.24 \\
\hline Control & 0.32 & & & & & Recessive & $1.64(092-2.94)$ & 0.09 \\
\hline Cases & 0.023 & & & & & Over-dominant & $0.69(0.49-0.96)$ & $0.02 *$ \\
\hline IL18 $105 \mathrm{~A}>\mathrm{T}$ & AA & AT & TT & $\mathrm{A} / \mathrm{T}$ & & & & \\
\hline Controls $(\mathrm{n}=300)$ & 46.7 & 42.0 & 11.3 & $0.68 / 0.32$ & 0.41 & Additive & $0.95(0.75-1.20)$ & 0.66 \\
\hline $\operatorname{Cases}(n=300)$ & 53.3 & 33.0 & $\begin{array}{l}13.7 \\
\text { OR } 0 .\end{array}$ & $\begin{array}{c}0.7 / 0.3 \\
0(0.70-1.15)\end{array}$ & & $\begin{array}{l}\text { Co-dominant } 1 \\
\text { Co-dominant2 }\end{array}$ & $\begin{array}{l}0.71(0.50-1.01) \\
1.13(0.68-1.90)\end{array}$ & 0.09 \\
\hline HWE(p) & & & & & & Dominant & $0.80(0.58-1.11)$ & 0.18 \\
\hline Control & 0.51 & & & & & Recessive & $1.31(0.80-2.15)$ & 0.28 \\
\hline Cases & 0.0021 & & & & & Over-dominant & $0.70(0.75-1.20)$ & $0.03 *$ \\
\hline
\end{tabular}

\subsection{Association of genotypes with Clinicopathological risk Factors of Breast cancer}

Analyses of association between genotypic and clinico-pathological features adjusted by age with multivariate logistic regression analyses in breast cancer patients revealed that the AA genotype of IL6 -597G $>$ A polymorphism was found to be associated with TNM staging $\left(\mathrm{T}_{2}-\mathrm{T}_{4}\right.$ late stage); GG genotype of IL6 -572C $>\mathrm{G}$ polymorphism was found to be significantly associated with late (advanced) stage of breast cancer patients. In addition, the frequency of AA genotype of IL6 -597G >A, GG genotype of IL6 -572C $>$ G and CC genotype of IL6 $-174 \mathrm{G}>\mathrm{C}$ gene polymorphism were found to be significantly associated with positive metastasis status in breast cancer patients as summarized in Table 2a. However, analysis of polymorphic variants $(-607 \mathrm{C}>\mathrm{A},-137 \mathrm{G}>\mathrm{C}$ and $105 \mathrm{~A}>\mathrm{T}$ ) of $I L 18$ gene polymorphisms with respect to histological subtype revealed that the AA and CA genotype of $-607 \mathrm{C}>\mathrm{A}$ polymorphism and $\mathrm{AT}+\mathrm{TT}$ genotype of $105 \mathrm{~A}>\mathrm{T}$ polymorphisms had significant association with lobular carcinoma subtype in breast cancer patients. Furthermore, CC and GC genotypes of $-137 \mathrm{G}>\mathrm{C}$ polymorphism was found to be significantly associated with $\mathrm{PgR}^{-\mathrm{ve}}$ and $\mathrm{HER} 2^{+\mathrm{ve}}$ breast cancer patients respectively (Table $2 b$ ). 
Table 2a: Genotype frequencies of $I L 6$ gene and clinicopathological features of breast cancer patients

\begin{tabular}{|c|c|c|c|c|c|c|c|c|c|}
\hline \multirow[t]{2}{*}{ CharacteristicsN=300(\%) } & \multicolumn{3}{|c|}{ IL6 -597G $>A$} & \multicolumn{3}{|c|}{ IL6 -572C $>\mathrm{G}$} & \multicolumn{3}{|c|}{ IL6 -174G $>C$} \\
\hline & GG & GA & $\mathbf{A A}$ & $\mathbf{C C}$ & CG & GG & GG & GC & $\mathbf{C C}$ \\
\hline \multicolumn{10}{|l|}{ Stage of the cancer } \\
\hline Early197 (65.67) & 53.8 & 31.0 & 15.2 & 25.9 & 65.0 & 9.1 & 33.5 & 42.1 & 24.1 \\
\hline Late103 (34.33) & 43.7 & 26.2 & 30.1 & 20.4 & 57.3 & 22.3 & 41.8 & 35.9 & 22.3 \\
\hline AOR (95\% CIs) & 1.0 (ref) & $1.05(0.59-.86)$ & $2.40(1.32-4.60)$ & 1.0 (ref) & $1.11(0.61-2.01)$ & $3.10(1.39-6.89)$ & $1.0(\mathrm{ref})$ & $0.68(0.40-1.18)$ & $0.74(0.39-1.39)$ \\
\hline$p$-value & \multicolumn{3}{|c|}{$0.012 *$} & \multicolumn{3}{|c|}{$0.008^{*}$} & \multicolumn{3}{|c|}{0.37} \\
\hline \multicolumn{10}{|l|}{ Type of the cancer } \\
\hline Ductal 247 (82.33) & 51.8 & 30.4 & 17.8 & 12.1 & 62.4 & 25.5 & 35.2 & 40.5 & 24.3 \\
\hline Lobular53 (17.67) & 43.4 & 24.5 & 32.1 & 20.8 & 62.3 & 16.9 & 41.5 & 37.7 & 20.8 \\
\hline $\operatorname{AOR}(95 \% \mathrm{CI})$ & 1.0 (ref) & $0.91(0.43-1.92)$ & $1.89(0.91-3.92)$ & 1.0 (ref) & $0.56(0.25-1.23)$ & $0.39(0.14-1.04)$ & $1.0(\mathrm{ref})$ & $0.80(0.41-1.57)$ & $0.75(0.34-1.68)$ \\
\hline$p$-value & \multicolumn{3}{|c|}{0.16} & \multicolumn{3}{|c|}{0.17} & \multicolumn{3}{|c|}{0.73} \\
\hline \multicolumn{10}{|l|}{ Axillary Lymph node Status } \\
\hline Negative 80 (26.67) & 45.0 & 28.8 & 26.2 & 31.2 & 48.0 & 8.8 & 32.5 & 37.5 & 30.0 \\
\hline Positive $220(73.33)$ & 52.26 & 29.54 & 18.2 & 21.4 & 63.2 & 15.4 & 37.7 & 40.9 & 21.4 \\
\hline AOR $(95 \% \mathrm{CI})$ & 1.0 (ref) & $0.88(0.48-1.62)$ & $0.59(0.31-1.15)$ & 1.0 (ref) & $1.54(0.85-2.76)$ & $2.58(1.02-6.06)$ & 1.0 (ref) & $0.94(0.51-1.12)$ & $0.61(0.31-1.18)$ \\
\hline$p$-value & \multicolumn{3}{|c|}{0.31} & \multirow{2}{*}{\multicolumn{3}{|c|}{0.11}} & \multicolumn{3}{|c|}{0.3} \\
\hline \multicolumn{7}{|l|}{ EgR Status } & & & \\
\hline Positive177 (59) & 49.7 & 29.9 & 20.4 & 22.6 & 64.4 & 13.0 & 32.5 & 37.5 & 30.0 \\
\hline Negative123 (41) & 51.2 & 28.5 & 20.3 & 26.0 & 59.4 & 14.6 & 37.7 & 40.9 & 21.4 \\
\hline$A O R(95 \% \mathrm{CI})$ & 1.0 (ref) & $0.92(0.54-1.58)$ & $0.97(0.52-1.80)$ & 1.0 (ref) & $0.80(0.46-1.39)$ & $0.98(0.45-2.12)$ & 1.0 (ref) & $0.94(0.51-1.72)$ & $0.61(0.31-1.18)$ \\
\hline$p$-value & \multicolumn{3}{|c|}{0.96} & \multicolumn{3}{|c|}{0.67} & \multicolumn{3}{|c|}{0.3} \\
\hline \multicolumn{10}{|l|}{ PgR Status } \\
\hline Positive 168 (56) & 50.59 & 30.35 & 19.06 & 20.2 & 66.7 & 13.1 & 36.9 & 42.3 & 20.8 \\
\hline Negative 132 (44) & 50.0 & 28.0 & 22.0 & 28.8 & 56.8 & 14.4 & 35.6 & 37.1 & 27.3 \\
\hline $\operatorname{AOR}(95 \% \mathrm{CI})$ & 1.0 (ref) & $0.93(0.55-1.59)$ & $1.17(0.64-2.15)$ & 1.0 (ref) & $1.08(0.67-1.76)$ & $0.55(0.211-1.41)$ & 1.0 (ref) & $0.91(0.54-1.54)$ & $1.36(0.75-2.48)$ \\
\hline$p$-value & \multicolumn{3}{|c|}{0.80} & \multicolumn{3}{|c|}{0.67} & \multicolumn{3}{|c|}{0.4} \\
\hline \multicolumn{10}{|l|}{ HER2/neu Status } \\
\hline Positive 163 (54.33) & 47.9 & 30.7 & 21.4 & 27.0 & 58.9 & 14.1 & 33.7 & 39.3 & 26.4 \\
\hline Negative 111 (37) & 55.9 & 27.9 & 16.2 & 20.7 & 64.9 & 14.4 & 39.6 & 39.6 & 20.8 \\
\hline AOR $(95 \% \mathrm{CI})$ & 1.0 (ref) & $0.78(0.45-1.36)$ & $0.65(0.33-1.25)$ & 1.0 (ref) & $1.43(0.80-2.59)$ & $1.33(0.59-3.00)$ & 1.0 (ref) & $0.85(0.49-1.47)$ & $0.67(0.35-1.27)$ \\
\hline p-value & \multicolumn{3}{|c|}{0.38} & & & 0.48 & & & 0.47 \\
\hline Metastasis Status & & & & & & & & & \\
\hline Positive 103 (34.33) & 49.8 & 33.0 & 17.2 & 26.9 & 63.5 & 9.6 & 30.5 & 43.5 & 25.9 \\
\hline Negative 197 (66.67 & 51.5 & 22.3 & 26.2 & 18.4 & 60.2 & 21.4 & 47.1 & 33.3 & 19.6 \\
\hline AOR $(95 \% \mathrm{CI})$ & 1.0 (ref) & $0.65(0.37-1.17)$ & $1.14(0.80-2.69)$ & 1.0 (ref) & $1.38(0.75-2.54)$ & $3.23(1.44-7.24)$ & 1.0 (ref) & $0.48(0.28-0.84)$ & $0.48(0.25-0.91)$ \\
\hline$p$-value & & & $0.06 \#$ & & & $0.01^{*}$ & & & $0.01 *$ \\
\hline
\end{tabular}


Table 2b: Genotype frequencies of $I L 18$ gene and clinicopathological features of breast cancer patients

\begin{tabular}{|c|c|c|c|c|c|c|c|c|c|}
\hline \multirow{2}{*}{ Characteristics N=300 (\%) } & \multicolumn{3}{|c|}{ IL18 -607C $>\mathrm{A}$} & \multicolumn{3}{|c|}{$I L-18-137 \mathrm{G}>\mathrm{C}$} & \multicolumn{3}{|c|}{$I L 18105 \mathrm{~A}>\mathrm{T}$} \\
\hline & $\mathbf{C C}$ & CA & $\overline{\mathbf{A A}}$ & GG & GC & $\mathrm{CC}$ & $\overline{\mathbf{A A}}$ & $\mathbf{A T}$ & TT \\
\hline \multicolumn{10}{|l|}{ Stage of the cancer } \\
\hline Early197 (65.67) & 54.8 & 37.1 & 8.1 & 57.9 & 33.0 & 9.1 & 53.8 & 30.5 & 15.7 \\
\hline Late103 (34.33) & 57.3 & 35.9 & 6.8 & 45.6 & 39.8 & 14.6 & 52.4 & 37.9 & 9.7 \\
\hline AOR (95\% CIs) & $1.0(\mathrm{ref})$ & $0.93(0.56-1.55)$ & $0.82(0.32-2.13)$ & 1.0 (ref) & $1.54(0.92-2.59)$ & $2.03(0.94-4.37)$ & 1.0 (ref) & $1.26(0.75-2.13)$ & $0.63(0.29-1.38)$ \\
\hline$p$-value & \multicolumn{3}{|c|}{0.9} & \multicolumn{3}{|c|}{0.09} & \multicolumn{3}{|c|}{0.22} \\
\hline \multicolumn{10}{|l|}{ Type of the cancer } \\
\hline Ductal 247 (82.33) & 59.1 & 35.2 & 5.7 & 54.7 & 36.0 & 9.3 & 56.3 & 32.0 & 11.7 \\
\hline Lobular53 (17.67) & 39.6 & 43.4 & 17.0 & 49.1 & 32.1 & 18.8 & 39.6 & 37.7 & 22.7 \\
\hline AOR (95\% CI) & 1.0 (ref) & $1.93(1.00-3.72)$ & $6.02(2.19-16.55)$ & 1.0 (ref) & $1.03(0.53-2.02)$ & $2.32(0.98-5.51)$ & 1.0 (ref) & $1.56(0.79-3.08)$ & $2.60(1.14-5.91)$ \\
\hline$p$-value & \multicolumn{3}{|c|}{$0.001^{*}$} & \multicolumn{3}{|c|}{0.16} & \multicolumn{3}{|c|}{$0.07 \#$} \\
\hline \multicolumn{10}{|l|}{ Axillary Lymph node Status } \\
\hline Negative $80(26.67)$ & 51.2 & 40.0 & 8.8 & 60.0 & 32.5 & 7.5 & 52.5 & 38.8 & 8.8 \\
\hline Positive 220 (73.33) & 57.3 & 35.5 & 7.2 & 51.4 & 36.4 & 12.2 & 53.6 & 30.9 & 15.5 \\
\hline AOR (95\% CI) & $1.0(\mathrm{ref})$ & $0.79(0.46-1.36)$ & $0.72(0.28-1.91)$ & 1.0 (ref) & $1.30(0.75-2.28)$ & $1.91(0.74-4.92)$ & 1.0 (ref) & $0.79(0.45-1.37)$ & $1.74(0.71-4.23)$ \\
\hline$p$-value & \multicolumn{3}{|c|}{0.63} & \multirow{2}{*}{\multicolumn{3}{|c|}{0.31}} & \multicolumn{3}{|c|}{0.2} \\
\hline \multicolumn{7}{|l|}{ EgR Status } & & & \\
\hline Positive177 (59) & 56.5 & 35.6 & 7.9 & 53.1 & 37.3 & 9.6 & 51.4 & 36.2 & 12.4 \\
\hline Negative123 (41) & 54.5 & 38.2 & 7.3 & 54.5 & 32.5 & 13.0 & 56.1 & 28.5 & 15.4 \\
\hline AOR (95\% CI) & 1.0 (ref) & $1.11(0.68-1.81)$ & $0.95(0.39-2.36)$ & 1.0 (ref) & $0.85(0.51-1.40)$ & $1.32(0.62-2.80)$ & 1.0 (ref) & $0.72(0.43-1.21)$ & $1.14(0.57-2.27)$ \\
\hline$p$-value & \multicolumn{3}{|c|}{0.89} & \multicolumn{3}{|c|}{0.53} & \multicolumn{3}{|c|}{0.35} \\
\hline \multicolumn{10}{|l|}{ PgR Status } \\
\hline Positive 168 (56) & 55.4 & 35.1 & 9.5 & 50.6 & 40.5 & 8.9 & 52.4 & 34.5 & 13.1 \\
\hline Negative 132 (44) & 56.1 & 38.6 & 5.3 & 50.0 & 15.1 & 34.9 & 54.5 & 31.1 & 14.4 \\
\hline AOR (95\% CI) & 1.0 (ref) & $1.08(0.67-1.76)$ & $0.55(0.21-1.41)$ & 1.0 (ref) & $0.37(0.20-0.68)$ & $3.94(2.03-7.68)$ & 1.0 (ref) & $0.86(0.52-1.43)$ & $1.05(0.53-2.10)$ \\
\hline$p$-value & \multicolumn{3}{|c|}{0.36} & \multicolumn{3}{|c|}{$0.002 *$} & \multicolumn{3}{|c|}{0.8} \\
\hline \multicolumn{10}{|l|}{ HER2/neu Status } \\
\hline Positive 163 (54.33) & 55.2 & 39.9 & 4.9 & 63.2 & 28.2 & 8.6 & 55.8 & 34.4 & 9.8 \\
\hline Negative 111 (37) & 59.5 & 30.6 & 9.9 & 46.9 & 41.4 & 11.7 & 56.8 & 29.7 & 13.5 \\
\hline AOR (95\% CI) & 1.0 (ref) & $0.71(0.42-1.20)$ & $1.87(0.71-4.92)$ & 1.0 (ref) & $1.98(1.17-3.36)$ & $1.84(0.81-4.20)$ & 1.0 (ref) & $0.85(0.50-1.46)$ & $1.35(0.62-2.94)$ \\
\hline$p$-value & \multicolumn{3}{|c|}{0.13} & & & $0.02 *$ & & & 0.54 \\
\hline Metastasis Status & & & & & & & & & \\
\hline Positive 103 (34.33) & 55.3 & 37.6 & 7.1 & 61.4 & 29.9 & 8.7 & 51.8 & 33.0 & 15.2 \\
\hline Negative 197 (66.67 & 56.3 & 35.0 & 8.7 & 38.8 & 45.6 & 15.6 & 56.3 & 33.0 & 10.7 \\
\hline AOR $(95 \%$ CI) & 1.0 (ref) & $0.91(0.55-1.52)$ & $1.21(0.49-2.96)$ & 1.0 (ref) & $2.41(1.43-4.07)$ & $2.85(1.32-6.15)$ & 1.0 (ref) & $0.92(0.54-1.56)$ & $0.64(0.30-1.38)$ \\
\hline$p$-value & & & 0.83 & & & $0.001 *$ & & & 0.52 \\
\hline
\end{tabular}


*p $<0.05$ is considered to be statistically significant; \#Borderline significant. The odds ratios (ORs) with their 95\% confidence intervals (CIs) were estimated by logistic regression models. The adjusted odds ratios (AORs) with their 95\% confidence intervals (CIs) were estimated by multiple logistic regression models, after controlling for age, (Estrogen receptor (EgR), Progesterone receptor (PgR), Human epidermal growth factor receptor 2/neu receptor (HER2/neu).

\subsection{HapMap analysis of different ethnic groups:}

We have compared the allele frequencies of IL6 and IL18 in different ethnic groups (Asian-JPT, AsianHCB, Japanese-JPT, and European CEU) using HapMap dataset and found that the frequency of G allele of -597, the $\mathrm{C}$ allele of -572 and $\mathrm{C}$ allele of -174 ; $\mathrm{C}$ allele of $-607, \mathrm{G}$ allele of -137 and A allele of 105 were similar to that of our population (Fig. 2).

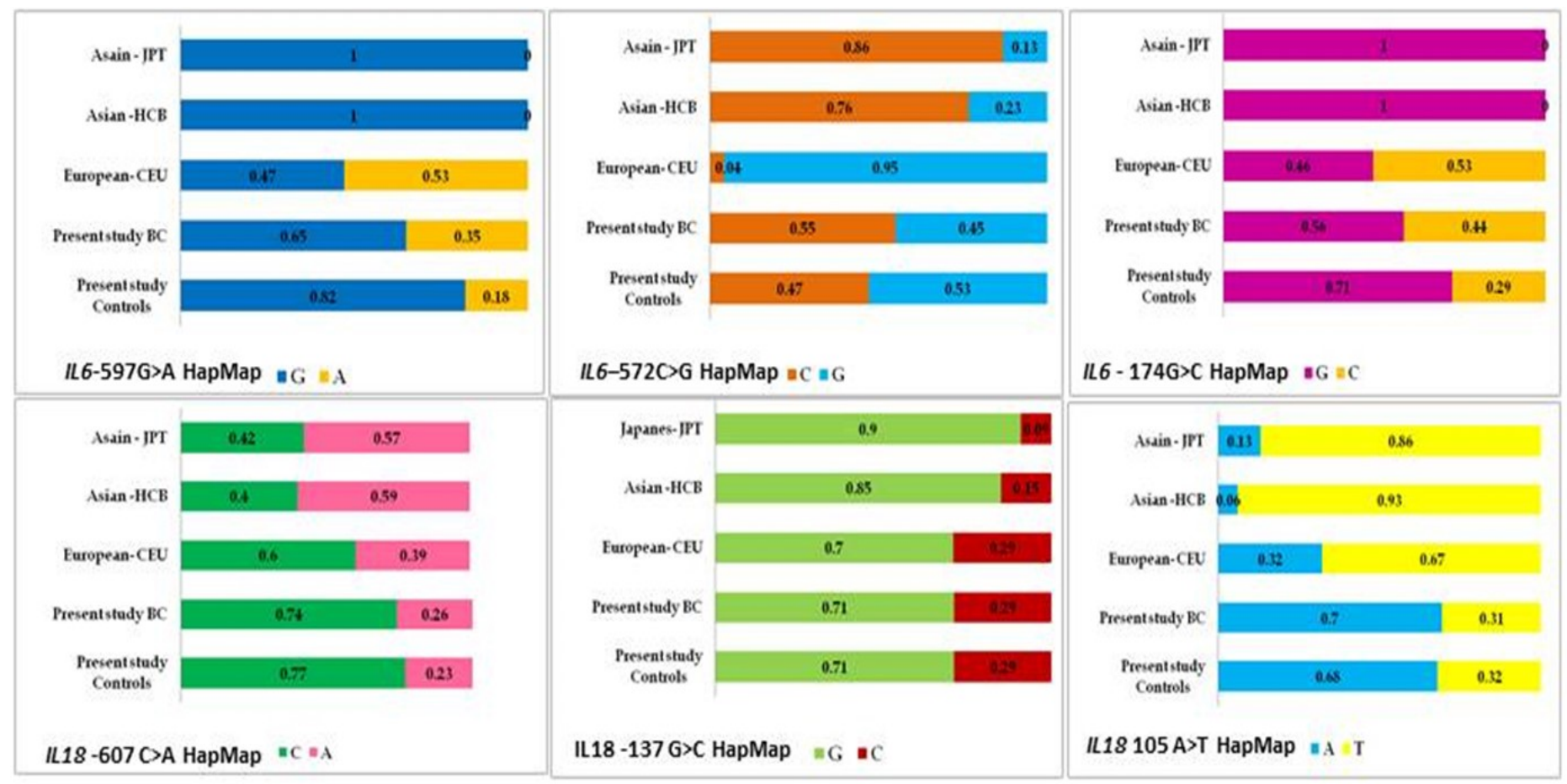

Figure 2. HapMap analysis of IL6 $-597 \mathrm{G}>\mathrm{A},-572 \mathrm{C}>\mathrm{G},-174 \mathrm{G}>\mathrm{C}$ and IL18 $(-607 \mathrm{C}>\mathrm{A},-137 \mathrm{G}>\mathrm{C}, 105 \mathrm{~A}>\mathrm{T})$ polymorphisms in Breast cancer

\subsection{Haplotype Analysis and Estimation of Linkage Disequilibrium (LD)}

IL6 haplotype: The analysis of IL6 gene showed eight haplotype groups among which G-G-G haplotype was most frequent, hence was chosen as a reference. Three haplotype (G-C-C, A-G-G and A-C-C) combinations had conferred 2.09, 2.25 and 4.72 folds risk for breast cancer respectively $(p<0.05)$, while G-C-G, G-G-C and A-G$\mathrm{C}$ haplotypes had conferred protection against breast cancer $(p<0.05)$ (Table 3$)$. Analysis of clinicopathological variables for haplotypes did not show any significant association with breast cancer (Table 4). 
Table 3: Frequencies of IL6 Haplotypes (-597 G>A, -572 C>G and -174 G>C) in healthy controls and breast cancer patients

\begin{tabular}{lllll}
\hline $\begin{array}{l}\text { Possible } \\
\text { haplotype }\end{array}$ & $\begin{array}{l}\text { Controls } \\
\text { No (\%) }\end{array}$ & $\begin{array}{l}\text { Patients } \\
\text { No (\%) }\end{array}$ & $\begin{array}{l}\text { Adjusted } \\
\text { OR(95\%CI) }\end{array}$ & $\boldsymbol{p}$-value \\
\hline GGG & 0.317 & 0.287 & $1.0($ ref $)$ & \\
GCC & 0.112 & 0.251 & $2.09(0.134-3.24)$ & $0.0011^{*}$ \\
GCG & 0.264 & 0.095 & $0.41(0.25-0.68)$ & $<0.001^{*}$ \\
AGG & 0.053 & 0.145 & $2.25(1.23-4.12)$ & $<0.0086^{*}$ \\
ACC & 0.014 & 0.164 & $4.72(2.30-9.67)$ & $<0.0001^{*}$ \\
GGC & 0.130 & 0.009 & $0.07(0.03-0.20)$ & $<0.0001$ \\
ACG & 0.076 & 0.034 & $0.61(0.32-1.16)$ & 0.13 \\
\hline AGC & 0.030 & 0.005 & $0.26(0.07-0.99)$ & 0.048 \\
\hline
\end{tabular}

Global haplotype association $p$-value: $<0.0001^{*}$, Adjusted (for age); OR and 95\%CI. $p<0.05$ significance

Table 4: Frequencies of IL-6 Haplotypes (-597 G>A, -572 C>G -174 G>C) and clinicopathological variable of breast cancer patients

\begin{tabular}{llllll}
\hline Clinicopathological Variables & Haplotype & \multicolumn{2}{l}{ Variable presence } & $\begin{array}{l}\text { Adjusted } \\
\text { OR(95\%CI) }\end{array}$ & $p$ - value \\
\hline Stage of the cancer & & Early State & Late Stage & & \\
& GCC & 2.96 & 0.184 & $0.62(0.34-1.12)$ & 0.12 \\
Type of the cancer & ACC & 0.154 & 0.180 & $0.99(0.58-1.70)$ & 0.98 \\
& & Ductal & Lobular & & \\
& GCC & 0.276 & 0.175 & $0.62(0.29-1.31)$ & 0.21 \\
Axillary Lymph node Status & ACC & 0.157 & 0.189 & $0.90(0.47-1.71)$ & 0.74 \\
& & Positive & Negative & & \\
& GCC & 0.263 & 0.257 & $0.70(0.37-1.37)$ & 0.26 \\
EgR Status & ACC & 0.217 & 0.141 & $0.52(0.29-0.92)$ & 0.025 \\
& & Positive & Negative & & \\
PgR Status & GCC & 0.236 & 0.288 & $1.15(0.68-1.95)$ & 0.61 \\
& ACC & 0.169 & 0.153 & $0.91(0.54-1.53)$ & 0.73 \\
HER2/neu Status & & Positive & Negative & & \\
& GCC & 0.243 & 0.277 & $1.25(0.73-2.12)$ & 0.41 \\
& ACC & 0.154 & 0.172 & $1.22(0.73-2.02)$ & 0.45 \\
Metastasis Status) & & Positive & Negative & & \\
& GCC & 0.245 & 0.296 & $1.05(0.61-1.79)$ & 0.87 \\
& ACC & 0.204 & 0.097 & $0.56(0.32-1.01)$ & 0.054 \\
\hline
\end{tabular}

Global haplotype association $p$-value: $<0.0001^{*}$, Adjusted (for age); OR and 95\%CI. $p<0.05$ significance

IL18 haplotype: None of the haplotypes of IL18 gene revealed any association with Breast cancer (Table 5). However, the analysis of clinicopathological variables with haplotypes revealed that A-G-A and A-C-T haplotypes to be associated with histological type of breast cancer (ductal) $(p<0.019)$. In addition, we also found strong association of C-C-T haplotype with axillary lymph node and HER2/neu receptor status while C-C-A with metastasis status respectively as shown in Table 6 . 
Table 5: Frequencies of $I L 18$ Haplotypes $(-607 \mathrm{C}>\mathrm{A},-137 \mathrm{G}>\mathrm{C}$ and $105 \mathrm{~A}>\mathrm{T})$ in healthy controls and breast cancer patients

\begin{tabular}{lllll}
\hline Possible haplotype & $\begin{array}{l}\text { Controls } \\
\text { No (\%) }\end{array}$ & $\begin{array}{l}\text { Patients } \\
\text { No (\%) }\end{array}$ & $\begin{array}{l}\text { Adjusted } \\
\text { OR(95\%CI) }\end{array}$ & $\boldsymbol{p}$-value \\
\hline CGA & 0.406 & 0.37 & $1.00($ ref $)$ & -- \\
CGT & 0.185 & 0.169 & $0.98(0.66-1.46)$ & 0.94 \\
CCA & 0.117 & 0.144 & $1.31(0.83-2.07)$ & 0.25 \\
AGA & 0.084 & 0.124 & $1.51(0.90-2.52)$ & 0.12 \\
ACA & 0.069 & 0.599 & $0.97(0.54-1.73)$ & 0.91 \\
CCT & 0.059 & 0.056 & $0.08(0.59-1.97)$ & 0.82 \\
AGT & 0.035 & 0.050 & $0.65(0.83-3.27)$ & 0.15 \\
ACT & 0.042 & 0.025 & $0.64(0.28-1.46)$ & 0.29 \\
\hline
\end{tabular}

Global haplotype association $p$-value: $<0.0001^{*}$, Adjusted (for age); OR and $95 \%$ CI. $p<0.05$ significance

Table 6: Frequencies of IL18 Haplotypes (-607 C >A, -137G $>C$ 105A $>$ T) and clinicopathological variables of breast cancer patients

\begin{tabular}{|c|c|c|c|c|c|}
\hline \multirow{2}{*}{$\begin{array}{l}\text { Clinicopathological Variables } \\
\text { Stage of the cancer }\end{array}$} & \multirow{2}{*}{$\begin{array}{l}\text { Haplotype } \\
\text { CCA }\end{array}$} & \multicolumn{2}{|c|}{ Variable presence } & \multirow{2}{*}{$\begin{array}{l}\begin{array}{l}\text { Adjusted } \\
\text { OR(95\%CI) }\end{array} \\
1.54(0.84-2.81)\end{array}$} & \multirow{2}{*}{$\begin{array}{c}p \text {-valuc } \\
0.16\end{array}$} \\
\hline & & $\begin{array}{l}\text { Early State } \\
0.144\end{array}$ & $\begin{array}{l}\text { Late Stage } \\
0.129\end{array}$ & & \\
\hline Type of the cancer & $\begin{array}{l}\text { AGA } \\
\text { ACT }\end{array}$ & $\begin{array}{l}\text { Ductal } \\
0.115 \\
0.041\end{array}$ & $\begin{array}{l}\text { Lobular } \\
0.152 \\
0.87\end{array}$ & $\begin{array}{l}3.00(1.20-7.49) \\
18.90(3.51-101.71)\end{array}$ & $\begin{array}{l}0.019^{*} \\
0.001^{*}\end{array}$ \\
\hline Axillary Lymph node Status & CCT & $\begin{array}{l}\text { Positive } \\
0.056\end{array}$ & $\begin{array}{l}\text { Negative } \\
0.009\end{array}$ & $7.82(1.03-59.29)$ & $0.04 *$ \\
\hline EgR Status & CCT & $\begin{array}{l}\text { Positive } \\
0.052\end{array}$ & $\begin{array}{l}\text { Negative } \\
0.063\end{array}$ & $1.30(0.59-2.86)$ & 0.52 \\
\hline PgR Status & СCT & $\begin{array}{l}\text { Positive } \\
0.047\end{array}$ & $\begin{array}{l}\text { Negative } \\
0.065\end{array}$ & $1.31(0.59-2.93)$ & 0.51 \\
\hline HER2/neu Status & CCT & $\begin{array}{l}\text { Positive } \\
0.027\end{array}$ & $\begin{array}{l}\text { Negative } \\
0.072\end{array}$ & $2.77(1.04-3.70)$ & $0.02 *$ \\
\hline Metastasis Status) & $\begin{array}{l}\text { CCA } \\
\text { CCT }\end{array}$ & $\begin{array}{l}\text { Positive } \\
0.109 \\
0.056 \\
\end{array}$ & $\begin{array}{l}\text { Negative } \\
0.210 \\
0.056 \\
\end{array}$ & $\begin{array}{l}2.00(1.08-3.70) \\
1.03(0.43-2.47) \\
\end{array}$ & $\begin{array}{l}0.027 * \\
0.94\end{array}$ \\
\hline
\end{tabular}

Global haplotype association $p$-value: $<0.0001^{*}$, Adjusted (for age); OR and 95\%CI. $p<0.05$ significance

Abbreviations: $\mathrm{CI}=$ confidence interval; $\mathrm{OR}=$ odds ratio; $\mathrm{SNP}=$ single nucleotide polymorphism.

\section{Linkage disequilibrium (LD) of SNPs}

The SNP IL6 -597G >A was in strong LD with IL6 -572C $>\mathrm{G}, I L 6-174 \mathrm{G}>\mathrm{C}, I L 18-607 \mathrm{C}>\mathrm{A}$ and $I L 18-137 \mathrm{G}>\mathrm{C}$. While, the SNPs of IL6 -572 C > G, IL6 -174G $>\mathrm{C}$ also showed a strong LD with IL18 $-607 \mathrm{C}>\mathrm{A}$ and IL18 $-137 \mathrm{G}$ $>$ C. Similarly, the SNPs IL18 -607C >A and IL18 -137G >C have also shown a strong LD with $105 \mathrm{~A}>\mathrm{T}$ with Pearson's correlation coefficient (D) of in red colour Table 7. 
Table 7. Linkage disequilibrium analysis between the Six Interleukin SNPs analyzed in Breast cancer patients.

\begin{tabular}{|l|l|l|l|l|l|}
\hline SNPs & IL6-597G $>$ A & IL6-572C $>$ G & IL6-174G $>$ C & IL18-607C $>$ A & IL18-137G $>$ C \\
\hline IL6-597G $>$ A & - & - & - & - & - \\
\hline IL6-572C $>$ G & 1.0 & - & - & - & - \\
\hline IL6 -174G $>$ C & 1.0 & 0.971 & - & - & - \\
\hline IL18-607C $>$ A & 1.0 & 1.0 & 1.0 & - & - \\
\hline IL18-137G $>$ C & 1.0 & 1.0 & 1.0 & 0.928 & - \\
\hline IL18 105A $>$ T & 0.974 & 0.953 & 0.978 & 1.0 & 1.0 \\
\hline
\end{tabular}

$\mathrm{D}$ values 1.0 (high grade of linkage disequilibrium) in red; $\mathrm{D}^{1}$ values $\mathrm{D}^{1}<1.0$ in yellow.

\subsection{Cox regression analysis of SNPs}

Multivariable Cox regression analysis showed that the adjusted risk of primary endpoint for breast cancer patients with GC of IL6 -174G >C polymorphism was 2.19-folds and GC heterozygote and CC homozygote of IL18 $-137 \mathrm{G}>\mathrm{C}$ gene polymorphisms to be 14.29 and 9.55 folds higher risk than non-carriers $(p<0.01)$. The study of COX regression analysis showed a decreased survival frequency in patients with breast cancer with AT heterozygote and TT homozygote of IL18 105A $>$ T gene polymorphism ( $p<0.05)$ (Table 8$)$.

Table 8: Cox regression analysis of SNPs for $I L 6(-597 \mathrm{G}>\mathrm{C},-572 \mathrm{C}>\mathrm{G}$ and $-174 \mathrm{G}>\mathrm{C})$ and $I L 18(-607,-137 \mathrm{G}>\mathrm{C}$ and $105 \mathrm{~A}>\mathrm{T}$ ) studied on 5 years median survival rate in breast cancer patients

\begin{tabular}{|c|c|c|c|}
\hline Polymorphism & $\begin{array}{l}\text { Genotypes } \\
\text { (5 years survival) }\end{array}$ & $\mathrm{HR}^{\mathrm{a}}(95 \% \mathrm{CI})$ & $p$-value \\
\hline \multirow{3}{*}{ IL6 -597G $>\mathrm{A}$} & $\mathrm{GG}(\mathrm{n}=122)$ & 1.00 (ref) & -- \\
\hline & $\mathrm{GC}(\mathrm{n}=75)$ & $0.67(0.39-1.12)$ & 0.13 \\
\hline & $\mathrm{CC}(\mathrm{n}=49)$ & $0.45(0.23-0.78)$ & 0.19 \\
\hline \multirow{3}{*}{ IL6 $-572 \mathrm{C}>\mathrm{G}$} & $\mathrm{CC}(\mathrm{n}=53)$ & $1.00($ ref $)$ & -- \\
\hline & $\mathrm{CG}(\mathrm{n}=157)$ & $1.82(0.92-3.58)$ & 0.81 \\
\hline & GG $(n=36)$ & $2.06(0.92-3.58)$ & 0.77 \\
\hline \multirow{3}{*}{ IL6 -174G $>\mathrm{C}$} & GG (n=89) & 1.00 (ref) & -- \\
\hline & $\mathrm{GC}(\mathrm{n}=103)$ & $2.19(1.15-4.18)$ & $0.017 *$ \\
\hline & $\mathrm{CC}(\mathrm{n}=54)$ & $1.20(0.61-2.34)$ & 0.58 \\
\hline \multirow{3}{*}{ IL $18-607 \mathrm{C}>\mathrm{A}$} & $\mathrm{CC}(\mathrm{n}=135)$ & 1.00 (ref) & -- \\
\hline & $\mathrm{CA}(\mathrm{n}=89)$ & $0.76(0.35-1.64)$ & 0.47 \\
\hline & $\mathrm{AA}(\mathrm{n}=22)$ & $1.12(0.21-2.43)$ & 0.58 \\
\hline \multirow{3}{*}{ IL18 $-137 \mathrm{G}>\mathrm{C}$} & $\mathrm{GG}(\mathrm{n}=126)$ & 1.00 (ref) & -- \\
\hline & $\mathrm{GC}(\mathrm{n}=88)$ & $14.29(6.25-31.30)$ & $0.01 *$ \\
\hline & $\mathrm{CC}(\mathrm{n}=32)$ & $9.45(3.75-23.81)$ & $0.01 *$ \\
\hline \multirow{3}{*}{ ILI8 $105 \mathrm{~A}>\mathrm{T}$} & AA $(n=141)$ & 1.00 (ref) & -- \\
\hline & $\operatorname{AT}(\mathrm{n}=71)$ & $0.59(0.34-1.02)$ & $0.05 *$ \\
\hline & $\mathrm{TT}(\mathrm{n}=34)$ & $0.51(0.24-1.07)$ & $0.05 *$ \\
\hline
\end{tabular}

*Significant at $p<0.05$, a-Hazardous Risk 
3.7 Kaplan-Meier 5 year's survival analysis of Breast Cancer Patients with respect to IL6 and ILI8 gene polymorphisms

Furthermore, Kaplan Meier analysis was carried out to know the influence of the genotypes on 5 years median survival rate, and the results showed that the patients with GA heterozygote of IL6 -597G>A, and TT homozygote of IL18 105A>T, polymorphism has borderline significant association and CC homozygote of IL6 $174 \mathrm{G}>\mathrm{C}$ and $\mathrm{CC}$ homozygote of $I L 18-137 \mathrm{G}>\mathrm{C}$ polymorphism has reduced median survival rate compared to that of GG homozygotes $(p<0.05)$ (Table 9 and Fig 3 A-F).

Table 9: Kaplan-Meier 5 years survival analysis of Breast Cancer Patients with respect to IL6 and IL18 gene polymorphisms

\begin{tabular}{lllllll}
\hline Variable & N $(\%)$ & Death $(\mathbf{n} \%)$ & HR(95\%CI) & Mean \pm SEM & Median & $p$-value \\
\hline IL6 $(-597 \mathrm{G}>$ A) & & & & & & \\
GG & $122(49.60)$ & $48(39.34)$ & $1.00(\mathrm{ref})$ & $50.47 \pm 1.56$ & 57.0 & \\
GA & $75(30.48)$ & $20(26.66)$ & & $55.06 \pm 1.52$ & 39.0 & $0.08 \#$ \\
AA & $49(19.91)$ & $11(22.44)$ & & $57.54 \pm 1.22$ & 51.0 & \\
IL6 $(-572 \mathrm{C}>\mathrm{G})$ & & & & & \\
CC & $53(21.54)$ & $10(18.86)$ & $1.00(\mathrm{ref})$ & $56.06 \pm 1.76$ & 52.0 & \\
CG & $157(63.82)$ & $54(34.39)$ & & $52.34 \pm 1.24$ & 57.0 & 0.13 \\
GG & $36(14.63)$ & $15(41.66)$ & & $53.26 \pm 2.52$ & 50.0 & \\
IL6 $(-174 \mathrm{G}>\mathrm{C})$ & & & & & \\
GG & $89(36.17)$ & $40(44.94)$ & $1.00(\mathrm{ref})$ & $49.84 \pm 1.79$ & 46.0 & \\
GC & $103(41.86)$ & $27(26.21)$ & & $54.69 \pm 1.37$ & 57.0 & \\
CC & $54(21.95)$ & $12(22.22)$ & & $56.41 \pm 1.66$ & 52.0 & $0.006^{*}$ \\
IL18(-607 C>A) & & & & & \\
CC & $135(54.87)$ & $37(27.40)$ & $1.00(\mathrm{ref})$ & $54.87 \pm 1.09$ & 57.0 & \\
CA & $89(36.17)$ & $34(38.20)$ & & $50.77 \pm 1.85$ & 48.0 & 0.26 \\
AA & $22(8.94)$ & $8(36.36)$ & & $53.77 \pm 1.85$ & 30.0 & \\
IL18 $(-137 \mathrm{G}>\mathrm{C})$ & & & & & \\
GG & $126(51.21)$ & $7(5.55)$ & $1.00(\mathrm{ref})$ & $59.94 \pm 0.60$ & 45.0 & \\
GC & $88(35.77)$ & $59(67.04)$ & & $45.80 \pm 1.86$ & 54.0 & $0.001^{*}$ \\
CC & $32(13.00)$ & $13(40.62)$ & & $50.88 \pm 3.04$ & 44.0 & \\
IL18 $(105 \mathrm{~A}>$ T) & & & & & \\
AA & $141(57.31)$ & $54(38.29)$ & $1.00(\mathrm{ref})$ & $51.12 \pm 1.38$ & 57.0 & \\
AT & $71(28.86)$ & $17(23.94)$ & & $56.04 \pm 1.44$ & 56.0 & $0.07 \#$ \\
TT & $34(13.82)$ & $8(23.52)$ & & $56.31 \pm 2.24$ & 30.0 & \\
\hline
\end{tabular}

${ }^{a} \log \operatorname{Rank} p$ - values by mantle COX regression statistically significant at $p<0.05$, "\#border line significant,

(Mean \pm SEM -5 years survival in months) 

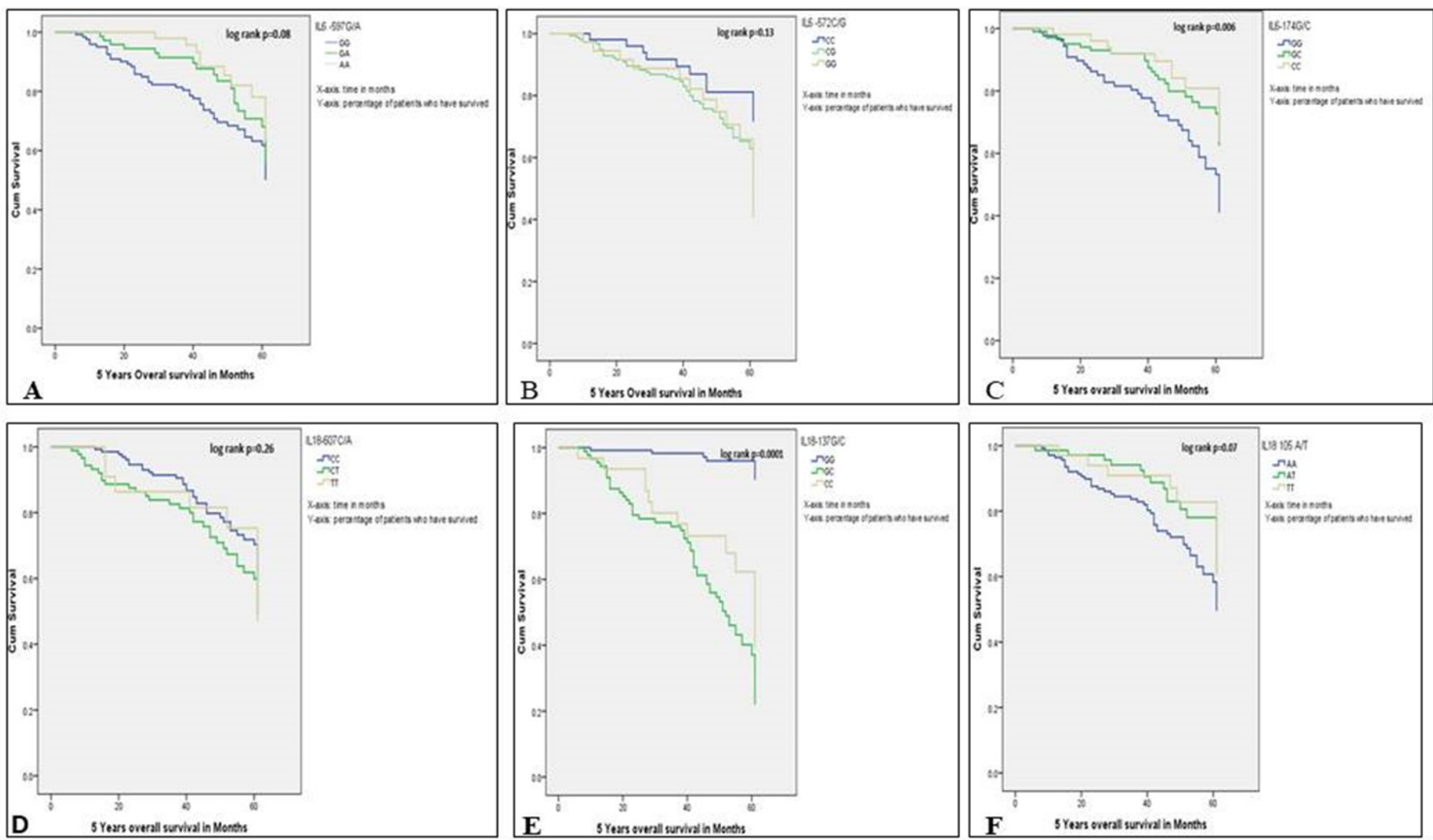

Figure 3. Kaplan-Meier Curves of 5-Year Survival among the Breast cancer patients: SNPs for A. IL6-597G>C; B. IL6 572C > G; C. IL6 -174G >C; D. IL18 -607C >A; E. IL18-137G >C and F. IL18 105 A > T gene polymorphisms.

\section{Transcription factor binding sites (TFBS) and Pre-mRNA secondary structures predictions}

The prediction of transcription factor binding sites (TFBSs) for IL6 gene (-597G>A, $-572 \mathrm{C}>\mathrm{G}$ and $174 \mathrm{G}>\mathrm{C}$ ) polymorphisms revealed that $\mathrm{G}$-allele of $-597 \mathrm{G}>\mathrm{A}$ polymorphism has NF-1, Sp1 and AP-2alph sites whereas A-allele has TBP, YY1 and NF-1. Further it is revealed that the C-allele of $-572 \mathrm{C}>\mathrm{G}$ polymorphism has binding site for Sp1, whereas G-allele has loss of Sp1 binding site. Furthermore, G allele of 174G $>\mathrm{C}$ polymorphism has no binding site while $\mathrm{C}$-allele has binding site for NF-1 respectively as depicted in Figure 4. Similarly, the IL18 gene $-607 \mathrm{C}>\mathrm{A}$ polymorphism has shown that $\mathrm{C}$ allele has binding sites for $\mathrm{C} / \mathrm{EBP}$ beta, MEB-1 sites whereas $\mathrm{A}$ allele has binding sites for C/EBP beta, HOXA4, MEB-1 sites. Further, the prediction of transcription factor binding sites of $-137 \mathrm{G}>\mathrm{C}$ polymorphism has shown that $\mathrm{G}$ allele has binding site for Nf-kappa $\mathrm{B}$ whereas $\mathrm{C}$ allele has an additional binding site for C/EBP beta and NF1 sites as respectively depicted in Fig. 4. The pre-mRNA secondary structures of IL18 105A $>$ T polymorphism had shown that T-allele $(-77.65 \mathrm{kcal} / \mathrm{mol})$ has higher entropy and is less stable than A-allele $(-76.08 \mathrm{kcal} / \mathrm{mol})$ as deduced from the minimum free energy was shown in Fig. 4. 

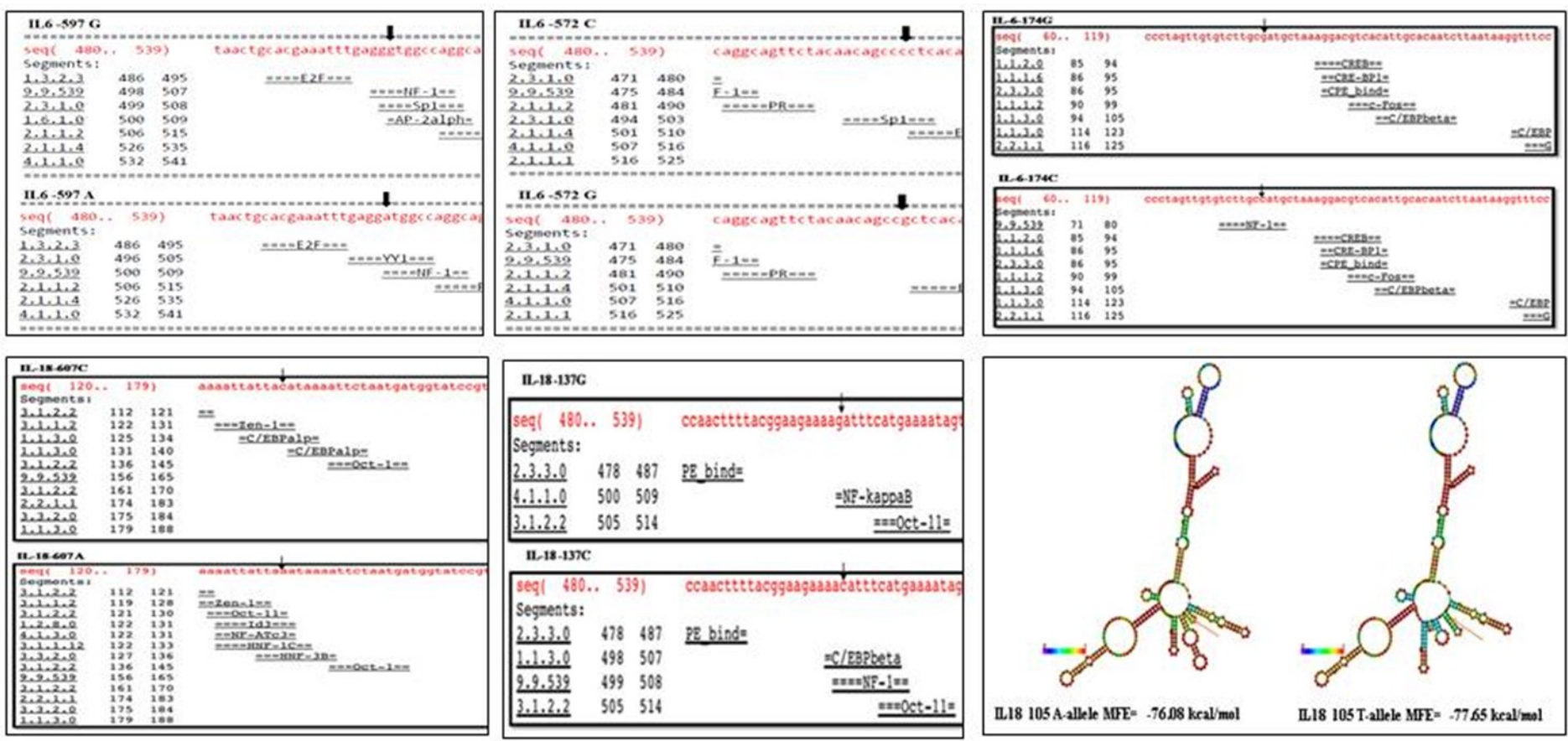

Figure 4 . Effect of the IL-6(-597G $>$ A, $-572 \mathrm{C}>\mathrm{G}$ and-174G $>\mathrm{C})$ and IL-18 $-607 \mathrm{C}>\mathrm{A}$, and $-137 \mathrm{G}>\mathrm{C}$ polymorphisms on transcription factor binding and IL-18 105A $>$ T Pre-mRNA secondary structure analysis respectively.

\section{Protein- protein interaction (PPI) analysis}

For protein interaction data, the present study utilized a human PPI dataset from the Search Tool for the Retrieval of Interacting genes/proteins. The obtained image contained several colored lines supporting their interactions, the more lines between two peptides and the more evidence to support their relationship. Our analysis revealed that the IL6 protein is found to be directly interacting with IL18, MMP1, 3, 9 and TIMP1 genes, while, $M M P 1$ and $M M P 9$ proteins are strongly interacting with each other and are co-expressed along with TIMP1 proteins as shown in Fig.5. 


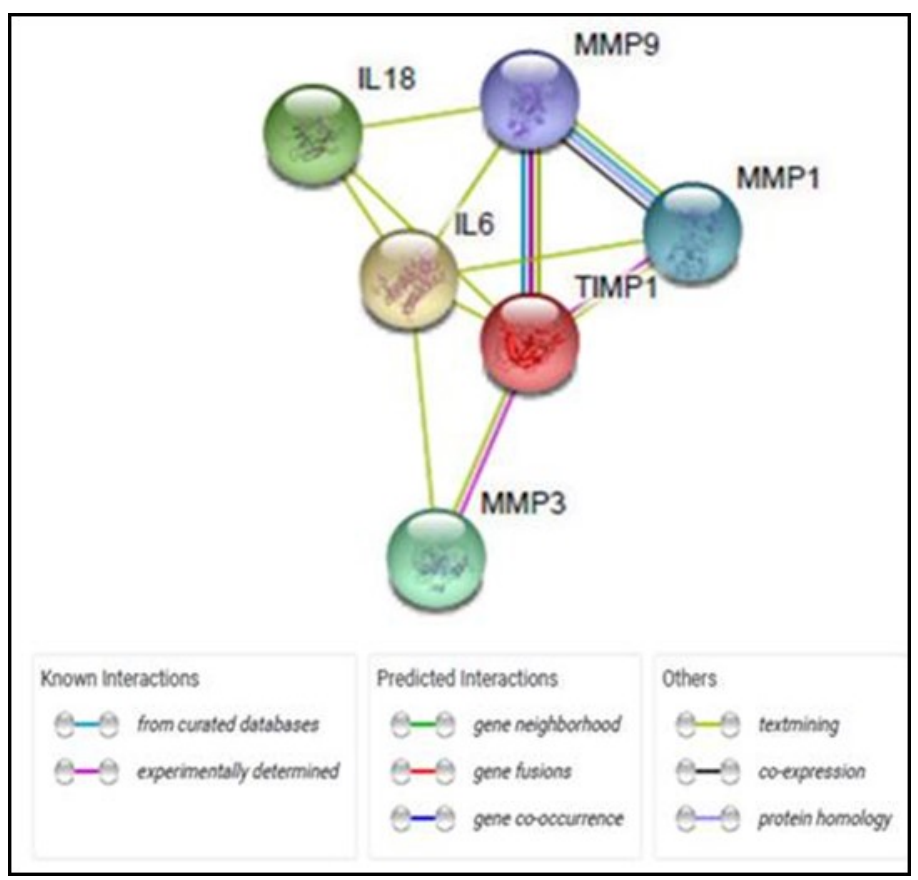

Fig.5 Protein -Protein interaction for IL6 and IL18 genes by String analysis.

\section{DISCUSSION}

Multiple molecular mechanisms are known to be involved in the development of breast cancer. Inflammation within the tumor microenvironment, one of the key mechanisms of carcinogenesis has been correlated with increased invasiveness and poor prognosis in many types of cancer including breast cancer [17]. Different cytokines are known to have diverse roles in breast cancer initiation and metastasis [18]. The cytokines are critical mediators of the inflammatory response [4]. The present study aimed to evaluate the association of IL6 (-597G>A, $572 \mathrm{C}>\mathrm{G},-174 \mathrm{G}>\mathrm{C})$ and $I L 18(-607 \mathrm{C}>\mathrm{A},-137 \mathrm{G}>\mathrm{C}, 105 \mathrm{~A}>\mathrm{T})$ gene polymorphic variants with the epidemiological and clinicopathological characteristics in south Indian Breast cancer patients. The genetic variations of IL6 gene are known to influence the transcriptional activity by affecting the expression, leading to the susceptibility and progression of breast cancer [19]. Three single-nucleotide polymorphisms $-597 \mathrm{G}>\mathrm{A},-572 \mathrm{C}>\mathrm{G}$ and $-174 \mathrm{G}>\mathrm{C}$ have been identified in the promoter region of the IL6 gene, which might have an effect on IL6 transcription and plasma IL6 levels in multiple human diseases [20].

IL6 $-597 \mathrm{G}>\mathrm{A}$ polymorphism was reported to be associated with serum IL6 levels and as a susceptibility factor in several diseases such as multiple myeloma [21], rheumatoid arthritis [22], Sepsis [23], cervical cancer [24] and chronic obstructive pulmonary disease [25]. The genotype and allele frequency distribution of IL6 -597G>A polymorphism in controls and breast cancer patients revealed that the AA genotype and A-allele frequency was higher in breast cancer patients compared to controls and has conferred 3.97 and 2.53 folds risk for breast cancer 
respectively. Several groups have shown an association between AA genotype IL6 -597G $>$ A polymorphism with elevated serum IL6 levels and incidence of chronic inflammation with poor survival in multiple cancers. IL6 $572 \mathrm{C}>\mathrm{G}$ polymorphism is another important promoter polymorphism associated with serum IL6 levels and susceptibility to several diseases like OSCC [26], hypertension [27], T2DM [28]. It has also been suggested that individuals carrying the C allele of IL6 $-572 \mathrm{C}>\mathrm{G}$ polymorphism have an increased risk for breast cancer. Similarly, the genotype and allele frequency distribution of $I L 6-572 \mathrm{C}>\mathrm{G}$ polymorphism in controls and breast cancer patients revealed that the $\mathrm{CG}$ and $\mathrm{GG}$ genotypes had a protective role against breast cancer. Further, G allele frequency was high in controls and was associated with reduced risk, indicating $\mathrm{C}$-allele carriers are at an increased risk for breast cancer.

Another functional polymorphism in the promoter region of the IL6 gene at position $-174 \mathrm{G}>\mathrm{C}$ has been reported to be associated with diseases, such as OSCC [26], Ovarian [29], Cervical and breast cancer [30,31]. Earlier studies by Lagmay et al., have shown that the CC genotype of IL6 promoter $-174 \mathrm{G}>\mathrm{C}$ polymorphism to be associated with elevated high-risk for neuroblastoma [32] and prostate cancer [33]. A recent study suggested that individuals carrying the $-174 \mathrm{C}$ allele have an elevated risk for breast cancer [34]. The genotype and allele frequency distribution of IL6 -174G $>$ C polymorphism in controls and breast cancer patients revealed that the frequency of CC genotype and $\mathrm{C}$ allele were significantly elevated in breast cancer cases and conferred 2.52 and 1.91 fold risk for breast cancer development respectively.

The $I L 18$ gene promoter $-607 \mathrm{C}>\mathrm{A}$ and $-137 \mathrm{G}>\mathrm{C}$ polymorphisms and $105 \mathrm{~A}>\mathrm{T}$ polymorphism in exon 4 are found to influence the expression and may affect the blood plasma level of IL18 [35]. IL18 gene (-607C $>\mathrm{A}$, $137 \mathrm{G}>\mathrm{C}$ and $105 \mathrm{~A}>\mathrm{T}$ ) polymorphisms have been associated with susceptibility to several diseases like CHD, Systemic lupus erythematosus (SLE), esophageal squamous cell carcinoma [36], prostate [37], colorectal [38], ovarian cancer [39], nasopharyngeal (rare type of head and neck) carcinoma [40] and breast cancer [41]. In contrary, no significant association was found when $I L 18-607 \mathrm{C}>\mathrm{A}, I L 18-137 \mathrm{G}>\mathrm{C}$ gene polymorphisms were studied in patients with Crohn disease or ulcerative colitis and other cancers. Recently, similar results were found in patients with oral cavity cancer where there was no significant association of IL18 -607C $>$ A polymorphism even after adjusting for age [42]. Similarly, in the present study the genotype and allele frequency distribution of IL18 $607 \mathrm{C}>\mathrm{A}$ polymorphism in controls and breast cancer patients revealed that there was no significant association of IL18 -607C $>$ A polymorphism with breast cancer.

Another single nucleotide polymorphism in the promoter region of the $I L 18$ gene at position $-137 \mathrm{G}>\mathrm{C}$ has been reported to be associated with a variety of diseases, such as Chronic Lymphocytic and Chronic Myelogenous [43], cervical [44], breast cancer [41] and unfavourable clinical outcome in patients affected by high-risk neuroblastoma, trauma inflammation etc [45]. In the present study, the genotype and allele frequency distribution of IL18 $-137 \mathrm{G}>\mathrm{C}$ polymorphism did not revealed any significant association with breast cancer. However, the frequency of CC genotype was slightly high in breast cancer patients compared to controls. The studies by Stassen et al., and Farjadfar et al., showed the relationship between IL18 gene exonic variants and predisposition to various 
malignant, viral and autoimmune diseases and experiments by have demonstrated that the $105 \mathrm{~A}>\mathrm{T}$ polymorphism of the IL18 gene may be associated with the pathogenesis of asthma in Japanese patients [46,47]. However in the present study there was no significancant association between IL18 105A $>$ T polymorphism and breast cancer in different genetic models studied.

Over expression of IL6 and IL18 genes have been found to be positively associated with the clinicopathological characteristics of several malignancies [26,48,49]. In the present study SNPs of IL6 (-597G $>$ A, $572 \mathrm{C}>\mathrm{G}$ and $174 \mathrm{G}>\mathrm{C})$ and $I L 18(607 \mathrm{C}>\mathrm{A}, 137 \mathrm{G}>\mathrm{C}$ and $105 \mathrm{~A}>\mathrm{T})$ genes were correlated with clinicopathological variables for their association in breast cancer progression and susceptibility. Our results revealed a significant association of AA genotype of IL6 -597 and GG genotype of IL6 -572 with TNM stage of breast cancer and CC genotype of IL6 -174 with metastasis, indicating the association of AA, GG and CC genotype with features of tumour phenotype in progression of breast cancer. The CA and AA genotypes of $607 \mathrm{C}>\mathrm{A}$ and AT \& TT of $105 \mathrm{~A}>\mathrm{T}$ of $I L 18$ polymorphisms, were found to be significantly associated with histological subtype (lobular) breast cancer. The GC and CC genotypes of $I L 18-137 \mathrm{G}>\mathrm{C}$ polymorphism were found to be significantly associated with PR, HER2/neu, and metastasis status. IL6 (-597G >A, $-572 \mathrm{C}>\mathrm{G}$, and $-174 \mathrm{G}>\mathrm{C})$ and IL18 (-607C $>\mathrm{A},-137 \mathrm{G}>\mathrm{C}$ and $105 \mathrm{~A}>\mathrm{T}$ ) SNP marker combinations exhibited complete LD. The present study revealed a strong association in each of the study groups.

The current available data suggest that the identification of patterns of genetic variations, in the form of haplotypes rather than single or point variations, may present a more promising approach [50-53]. In the present study haplotype analysis of IL6 gene polymorphisms revealed a significant association with risk for breast cancer development by 2.09 folds for G-C-C haplotype, 2.25 folds for A-G-G haplotype and 4.72 folds for A-C-C haplotype respectively, while G-C-G, G-G-C and A-G-C haplotypes conferred protection against breast cancer. The haplotype containing both risk alleles (A allele of $-597 \mathrm{G}>\mathrm{A}, \mathrm{G}$ allele of $-572 \mathrm{C}>\mathrm{G}$ and $\mathrm{C}$ allele of $-174 \mathrm{G}>\mathrm{C}$ ) conferred enhanced risk for breast cancer. These results are in agreement with individual SNP analysis. The analysis of haplotype groups of $I L 18$ gene polymorphisms did not revealed any significant association with breast cancer susceptibility but the combined effect of IL18 gene polymorphisms (-607C $>$ A, $-137 \mathrm{G}>\mathrm{C} 105 \mathrm{~A}>\mathrm{T}$ ) with clinicopathological variables of haplotypes showed a significant association with ductal and lobular carcinoma, Further, our results showed that the association of haplotypes with axillary lymph nodes (C-C-T), HER2neu (C-CT) and metastasis (C-C-A) status in breast cancer patients. In addition to above haplotype analysis we have also analysed the allele frequency distribution of IL6 -597G $>$ A, $572 \mathrm{C}>\mathrm{G}$ and $174 \mathrm{G}>\mathrm{C}$ and IL18-607C $>\mathrm{A}, 137 \mathrm{G}>\mathrm{C}$ and $105 \mathrm{~A}>\mathrm{T}$ in various populations along with Indians by HapMap and found that A allele of $-597, \mathrm{G}$ allele of $-572, \mathrm{C}$ allele of -174 , A allele of $-607, \mathrm{C}$ allele of -137 and $\mathrm{T}$ allele of 105 were significantly associated with $\mathrm{BC}$. In addition, the 5 years cox-regression survival analysis found a significant association with IL6 -174G $>$ C and IL18$137 \mathrm{G}>\mathrm{C}$ polymorphisms. The functional polymorphisms in IL6 and IL18 could lead to altered gene expression, consequently creating imbalance in the vital cytokines system that results in excessive tumor progression and breast 
cancer development. To our best knowledge, present study is first to report the combined effect of three polymorphisms and haplotypes (A-C-C, A-G-G and G-C-C) along with the clinicopathological parameters which showed a significant association of IL6 polymorphism with BC. Overall, our results revealed that the polymorphisms in the promoter region of IL6, and IL18 genes when correlated with clinicopathological characteristics and survival rate have shown significant effects on the risk and progression of breast cancer, substantiated by in-silico analysis.

\section{Conclusions}

The study is focussed to understand the clinical importance of IL6 and IL18 gene variants in progression of breast cancer and to help in identifying individuals at risk of developing breast cancer. Interleukins are considered as promising targets for therapy due to their strong involvement in tumor pathology and progression at both molecular and clinicopathological levels. Therefore, understanding cancer subtypes, functional epigenomics, transcriptomic programs and signalling pathways regulating $I L s$ expression is critical for developing breast cancer therapeutics, not only to treat but also to prevent cancer. Further such a comprehensive approach may reveal highly prominent candidate molecular markers in future for breast cancer diagnosis and prognosis.

Limitation of the study: To the best of our knowledge this is the first study reporting on the combined effects of SNPs of IL6 and IL18 genes in correlation with clinicopathological variables along with LD, survival rate and Insilico analysis. However, our study has several limitations. Firstly, a small study that was analysed in South Indian population, because we restricted the study subjects to individuals of South Indian ethnicity; it is uncertain whether these results can be generalized to other populations. Second, there were few patients with missing data on hormonal receptor status, which may bias the results indicating an association with advanced disease status. Third, our LD and survival analysis included only 600 samples and this may have limited the power of the pooled results. Therefore, collaborative studies on different populations are necessary to corroborate our findings.

\section{Author Contributions:}

Chiranjeevi Padala: Concept and design; provision of study materials, methodology; data collection; analysis; validation and interpretation; article writing review; editing and final approval of the article. Kaushik Puranam, Nivas Shyamala, Keerthi Kupusala and Ramanjaneyulu, Kishore kumar Gundapaneni Rajesh Kumar Galimudi, and Mohini Aiyengar Tupurani: Resources and writing-review and editing; Collection and assembly of data and methodology. Aparna Suryadevera and Sanjeeva kumari Chinta: Provision of BC patients' blood samples; data collection; analysis and interpretation. Surekha Rani Hanumanth: Concept and design, Supervision; Administrative support; data analysis and interpretation; article writing, editing, and final approval of the article.

Conflicts of Interest: The authors declare no conflicts of interest. 


\section{Acknowledgments}

We acknowledge the subjects and hospital technical staff for their participation in this study, also thank Prof. Vishnupriya Satti lab, Dept. of Genetics, Osmania University for their continuous support in critical analysis and methodology. We thank UGC-RGNF No.F.16-1784(SC)/2010(SA-III), and UGC-DR. S. Kothari Post-doctoral fellowship award No.F.4-2/2006 (BSR)/BL/17-18/0584; and UGC-MRP (UGC MRP-F.No.42-52/2013[SR]), \& OU-DST-PURSE-II, DST-FIST and CAS-II programme New Delhi, INDIA, for providing grants.

\section{REFERENCES}

1. Darwiche, N. Epigenetic mechanisms and the hallmarks of cancer: an intimate affair. Am J Cancer Res 2020, 10, 1954-1978.

2. Hosonaga, M.; Saya, H.; Arima, Y. Molecular and cellular mechanisms underlying brain metastasis of breast cancer. Cancer Metastasis Rev 2020, 39, 711-720, doi:10.1007/s10555-020-09881-y.

3. Esquivel-Velazquez, M.; Ostoa-Saloma, P.; Palacios-Arreola, M.I.; Nava-Castro, K.E.; Castro, J.I.; Morales-Montor, J. The role of cytokines in breast cancer development and progression. J Interferon Cytokine Res 2015, 35, 1-16, doi:10.1089/jir.2014.0026.

4. Kany, S.; Vollrath, J.T.; Relja, B. Cytokines in Inflammatory Disease. Int J Mol Sci 2019, 20, doi:10.3390/ijms20236008.

5. Al-Eitan, L.N.; Al-Ahmad, B.H.; Almomani, F.A. The Association of IL-1 and HRAS Gene Polymorphisms with Breast Cancer Susceptibility in a Jordanian Population of Arab Descent: A GenotypePhenotype Study. Cancers (Basel) 2020, 12, doi:10.3390/cancers12020283.

6. Jung, J.H.; Jeong, H.S.; Choi, S.J.; Song, G.G.; Kim, J.H.; Lee, T.H.; Han, Y. Associations between interleukin 18 gene polymorphisms and susceptibility to vasculitis: A meta-analysis. Sarcoidosis Vasc Diffuse Lung Dis 2020, 37, 203-211, doi:10.36141/svdld.v37i2.9399.

7. Yang, H.; Zhou, X.; Xu, D.; Chen, G. The IL-6 rs12700386 polymorphism is associated with an increased risk of developing osteoarthritis in the knee in the Chinese Han population: a case-control study. BMC Med Genet 2020, 21, 199, doi:10.1186/s12881-020-01139-2.

8. Howell, W.M.; Rose-Zerilli, M.J. Cytokine gene polymorphisms, cancer susceptibility, and prognosis. $J$ Nutr 2007, 137, 194S-199S, doi:10.1093/jn/137.1.194S.

9. Chen, L.; Deng, H.; Cui, H.; Fang, J.; Zuo, Z.; Deng, J.; Li, Y.; Wang, X.; Zhao, L. Inflammatory responses and inflammation-associated diseases in organs. Oncotarget 2018, 9, 7204-7218, doi:10.18632/oncotarget.23208. 
10. Velez, D.R.; Fortunato, S.J.; Williams, S.M.; Menon, R. Interleukin-6 (IL-6) and receptor (IL6-R) gene haplotypes associate with amniotic fluid protein concentrations in preterm birth. Hum Mol Genet 2008, 17, 1619-1630, doi:10.1093/hmg/ddn049.

11. Shyamala, N.; Gundapaneni, K.K.; Galimudi, R.K.; Tupurani, M.A.; Padala, C.; Puranam, K.; Kupsal, K.; Kummari, R.; Gantala, S.R.; Nallamala, K.R.; et al. PCSK9 genetic (rs11591147) and epigenetic (DNA methylation) modifications associated with PCSK9 expression and serum proteins in CAD patients. $J$ Gene Med 2021, e3346, doi:10.1002/jgm.3346.

12. Sole, X.; Guino, E.; Valls, J.; Iniesta, R.; Moreno, V. SNPStats: a web tool for the analysis of association studies. Bioinformatics 2006, 22, 1928-1929, doi:10.1093/bioinformatics/btl268.

13. Barrett, J.C.; Fry, B.; Maller, J.; Daly, M.J. Haploview: analysis and visualization of LD and haplotype maps. Bioinformatics 2005, 21, 263-265, doi:10.1093/bioinformatics/bth457.

14. Berkman, S.J.; Roscoe, E.M.; Bourret, J.C. Comparing self-directed methods for training staff to create graphs using Graphpad Prism. J Appl Behav Anal 2019, 52, 188-204, doi:10.1002/jaba.522.

15. Zuker, M.; Stiegler, P. Optimal computer folding of large RNA sequences using thermodynamics and auxiliary information. Nucleic Acids Res 1981, 9, 133-148, doi:10.1093/nar/9.1.133.

16. Szklarczyk, D.; Morris, J.H.; Cook, H.; Kuhn, M.; Wyder, S.; Simonovic, M.; Santos, A.; Doncheva, N.T.; Roth, A.; Bork, P.; et al. The STRING database in 2017: quality-controlled protein-protein association networks, made broadly accessible. Nucleic Acids Res 2017, 45, D362-D368, doi:10.1093/nar/gkw937.

17. Feng, Y.; Spezia, M.; Huang, S.; Yuan, C.; Zeng, Z.; Zhang, L.; Ji, X.; Liu, W.; Huang, B.; Luo, W.; et al. Breast cancer development and progression: Risk factors, cancer stem cells, signaling pathways, genomics, and molecular pathogenesis. Genes Dis 2018, 5, 77-106, doi:10.1016/j.gendis.2018.05.001.

18. Fasoulakis, Z.; Kolios, G.; Papamanolis, V.; Kontomanolis, E.N. Interleukins Associated with Breast Cancer. Cureus 2018, 10, e3549, doi:10.7759/cureus.3549.

19. Masjedi, A.; Hashemi, V.; Hojjat-Farsangi, M.; Ghalamfarsa, G.; Azizi, G.; Yousefi, M.; Jadidi-Niaragh, F. The significant role of interleukin- 6 and its signaling pathway in the immunopathogenesis and treatment of breast cancer. Biomed Pharmacother 2018, 108, 1415-1424, doi:10.1016/j.biopha.2018.09.177.

20. Tanaka, T.; Narazaki, M.; Kishimoto, T. IL-6 in inflammation, immunity, and disease. Cold Spring Harb Perspect Biol 2014, 6, a016295, doi:10.1101/cshperspect.a016295.

21. Shahzad, M.N.; Ijaz, I.; Naqvi, S.; Yan, C.; Lin, F.; Li, S.; Huang, C. Association between interleukin gene polymorphisms and multiple myeloma susceptibility. Mol Clin Oncol 2020, 12, 212-224, doi:10.3892/mco.2020.1979.

22. Favalli, E.G. Understanding the Role of Interleukin-6 (IL-6) in the Joint and Beyond: A Comprehensive Review of IL-6 Inhibition for the Management of Rheumatoid Arthritis. Rheumatol Ther 2020, 7, 473-516, doi:10.1007/s40744-020-00219-2. 
23. $\mathrm{Hu}, \mathrm{P}$.; Chen, Y.; Pang, J.; Chen, X. Association between IL-6 polymorphisms and sepsis. Innate Immun 2019, 25, 465-472, doi:10.1177/1753425919872818.

24. Harmer, D.; Falank, C.; Reagan, M.R. Interleukin-6 Interweaves the Bone Marrow Microenvironment, Bone Loss, and Multiple Myeloma. Front Endocrinol (Lausanne) 2018, 9, 788, doi:10.3389/fendo.2018.00788.

25. Kirtipal, N.; Thakur, H.; Sobti, R.C.; Janmeja, A.K. Association between IL6 gene polymorphism and the risk of chronic obstructive pulmonary disease in the north Indian population. Mol Biol Res Commun 2020, 9, 41-43, doi:10.22099/mbrc.2019.34594.1431.

26. Singh, P.K.; Chandra, G.; Bogra, J.; Gupta, R.; Kumar, V.; Jain, A.; Hussain, S.R.; Mahdi, A.A.; Ahmad, M.K. Association of interleukin-6 genetic polymorphisms with risk of OSCC in Indian population. Meta Gene 2015, 4, 142-151, doi:10.1016/j.mgene.2015.03.002.

27. Ma, H.; Sun, G.; Wang, W.; Zhou, Y.; Liu, D.; Tong, Y.; Lu, Z. Association Between Interleukin-6 -572 $\mathrm{C}>\mathrm{G}$ and $-174 \mathrm{G}>\mathrm{C}$ Polymorphisms and Hypertension: A Meta-analysis of Case-control Studies. Medicine (Baltimore) 2016, 95, e2416, doi:10.1097/MD.0000000000002416.

28. Saxena, M.; Agrawal, C.G.; Srivastava, N.; Banerjee, M. Interleukin-6 (IL-6)-597 A/G (rs1800797) \& -174 G/C (rs1800795) gene polymorphisms in type 2 diabetes. Indian J Med Res 2014, 140, 60-68.

29. Ding, D.C.; Liu, H.W.; Chu, T.Y. Interleukin-6 from Ovarian Mesenchymal Stem Cells Promotes Proliferation, Sphere and Colony Formation and Tumorigenesis of an Ovarian Cancer Cell Line SKOV3. $J$ Cancer 2016, 7, 1815-1823, doi:10.7150/jca.16116.

30. $\mathrm{Pu}, \mathrm{X}$.; Gu, Z.; Wang, X. Polymorphisms of the interleukin 6 gene and additional gene-gene interaction contribute to cervical cancer susceptibility in Eastern Chinese women. Arch Gynecol Obstet 2016, 294, 1305-1310, doi:10.1007/s00404-016-4175-X.

31. Duan, H.X.; Chen, Y.Y.; Shi, J.Z.; Ren, N.N.; Li, X.J. Association of IL-6 -174G>C (rs1800795) polymorphism with cervical cancer susceptibility. Biosci Rep 2018, 38, doi:10.1042/BSR20181071.

32. Lagmay, J.P.; London, W.B.; Gross, T.G.; Termuhlen, A.; Sullivan, N.; Axel, A.; Mundy, B.; Ranalli, M.; Canner, J.; McGrady, P.; et al. Prognostic significance of interleukin-6 single nucleotide polymorphism genotypes in neuroblastoma: rs1800795 (promoter) and rs8192284 (receptor). Clin Cancer Res 2009, 15, 5234-5239, doi:10.1158/1078-0432.CCR-08-2953.

33. Bharti, R.; Dey, G.; Ojha, P.K.; Rajput, S.; Jaganathan, S.K.; Sen, R.; Mandal, M. Diacerein-mediated inhibition of IL-6/IL-6R signaling induces apoptotic effects on breast cancer. Oncogene 2016, 35, 39653975, doi:10.1038/onc.2015.466.

34. Maas, P.; Barrdahl, M.; Joshi, A.D.; Auer, P.L.; Gaudet, M.M.; Milne, R.L.; Schumacher, F.R.; Anderson, W.F.; Check, D.; Chattopadhyay, S.; et al. Breast Cancer Risk From Modifiable and Nonmodifiable Risk Factors Among White Women in the United States. JAMA Oncol 2016, 2, 1295-1302, doi:10.1001/jamaoncol.2016.1025. 
35. Warchol, T.; Lianeri, M.; Wudarski, M.; Lacki, J.K.; Jagodzinski, P.P. IL-18 105 A>C polymorphism contributes to renal manifestations in patients with SLE. Rheumatol Int 2009, 30, 187-191, doi:10.1007/s00296-009-0934-3.

36. Wei, Y.S.; Lan, Y.; Liu, Y.G.; Tang, H.; Tang, R.G.; Wang, J.C. Interleukin-18 gene promoter polymorphisms and the risk of esophageal squamous cell carcinoma. Acta Oncol 2007, 46, 1090-1096, doi:10.1080/02841860701373595.

37. Liu, J.M.; Liu, J.N.; Wei, M.T.; He, Y.Z.; Zhou, Y.; Song, X.B.; Ying, B.W.; Huang, J. Effect of IL-18 gene promoter polymorphisms on prostate cancer occurrence and prognosis in Han Chinese population. Genet Mol Res 2013, 12, 820-829, doi:10.4238/2013.March.15.2.

38. Wang, M.; Zhu, X.Y.; Wang, L.; Lin, Y. The -607C/A polymorphisms in interleukin-18 gene promoter contributes to cancer risk: evidence from a meta-analysis of 22 case-control studies. PLoS One 2013, 8, e76915, doi:10.1371/journal.pone.0076915.

39. Palmieri, R.T.; Wilson, M.A.; Iversen, E.S.; Clyde, M.A.; Calingaert, B.; Moorman, P.G.; Poole, C.; Anderson, A.R.; Anderson, S.; Anton-Culver, H.; et al. Polymorphism in the IL18 gene and epithelial ovarian cancer in non-Hispanic white women. Cancer Epidemiol Biomarkers Prev 2008, 17, 3567-3572, doi:10.1158/1055-9965.EPI-08-0548.

40. Riedel, F.; Adam, S.; Feick, P.; Haas, S.; Gotte, K.; Hormann, K.; Mannheim Alcohol Study, G. Expression of IL-18 in patients with head and neck squamous cell carcinoma. Int J Mol Med 2004, 13, 267-272.

41. Back, L.K.; Farias, T.D.; da Cunha, P.A.; Muniz, Y.C.; Ribeiro, M.C.; Fernandes, B.L.; Fernandes, C.K.; de Souza, I.R. Functional polymorphisms of interleukin-18 gene and risk of breast cancer in a Brazilian population. Tissue Antigens 2014, 84, 229-233, doi:10.1111/tan.12367.

42. Tsai, H.T.; Hsin, C.H.; Hsieh, Y.H.; Tang, C.H.; Yang, S.F.; Lin, C.W.; Chen, M.K. Impact of interleukin18 polymorphisms $-607 \mathrm{~A} / \mathrm{C}$ and $-137 \mathrm{G} / \mathrm{C}$ on oral cancer occurrence and clinical progression. PLoS One 2013, 8, e83572, doi:10.1371/journal.pone.0083572.

43. Yalcin, S.; Mutlu, P.; Cetin, T.; Sarper, M.; Ozgur, G.; Avcu, F. The -137G/C Polymorphism in Interleukin18 Gene Promoter Contributes to Chronic Lymphocytic and Chronic Myelogenous Leukemia Risk in Turkish Patients. Turk J Haematol 2015, 32, 311-316, doi:10.4274/tjh.2014.0126.

44. Jia, Y.; Zang, A.; Jiao, S.; Chen, S.; Yan, F. The interleukin-18 gene promoter -607 A/C polymorphism contributes to non-small-cell lung cancer risk in a Chinese population. Onco Targets Ther 2016, 9, 17151719, doi:10.2147/OTT.S99581.

45. Stassen, N.A.; Breit, C.M.; Norfleet, L.A.; Polk, H.C., Jr. IL-18 promoter polymorphisms correlate with the development of post-injury sepsis. Surgery 2003, 134, 351-356, doi:10.1067/msy.2003.248.

46. Higa, S.; Hirano, T.; Mayumi, M.; Hiraoka, M.; Ohshima, Y.; Nambu, M.; Yamaguchi, E.; Hizawa, N.; Kondo, N.; Matsui, E.; et al. Association between interleukin-18 gene polymorphism 105A/C and asthma. Clin Exp Allergy 2003, 33, 1097-1102, doi:10.1046/j.1365-2222.2003.01739.x. 
47. Escarceller, M.; Buchwald, M.; Singleton, B.K.; Jeggo, P.A.; Jackson, S.P.; Moustacchi, E.; Papadopoulo, D. Fanconi anemia C gene product plays a role in the fidelity of blunt DNA end-joining. J Mol Biol 1998, 279, 375-385, doi:10.1006/jmbi.1998.1784.

48. Korobeinikova, E.; Ugenskiene, R.; Insodaite, R.; Rudzianskas, V.; Jaselske, E.; Poskiene, L.; Juozaityte, E. Association of angiogenesis and inflammation-related gene functional polymorphisms with early-stage breast cancer prognosis. Oncol Lett 2020, 19, 3687-3700, doi:10.3892/ol.2020.11521.

49. Aman, N.A.; Doukoure, B.; Koffi, K.D.; Koui, B.S.; Traore, Z.C.; Kouyate, M.; Effi, A.B. HER2 overexpression and correlation with other significant clinicopathologic parameters in Ivorian breast cancer women. BMC Clin Pathol 2019, 19, 1, doi:10.1186/s12907-018-0081-4.

50. International HapMap, C. A haplotype map of the human genome. Nature 2005, 437, 1299-1320, doi: $10.1038 /$ nature 04226.

51. Lee, Y.; Lee, H. Integrative reconstruction of cancer genome karyotypes using InfoGenomeR. Nat Commun 2021, 12, 2467, doi:10.1038/s41467-021-22671-6.

52. Browning, S.R.; Browning, B.L. Haplotype phasing: existing methods and new developments. Nat Rev Genet 2011, 12, 703-714, doi:10.1038/nrg3054.

53. Ahmad, N.; Ammar, A.; Storr, S.J.; Green, A.R.; Rakha, E.; Ellis, I.O.; Martin, S.G. IL-6 and IL-10 are associated with good prognosis in early stage invasive breast cancer patients. Cancer Immunol Immunother 2018, 67, 537-549, doi:10.1007/s00262-017-2106-8. 